\title{
Oral Delivery of a Synthetic Sterol Reduces Axonopathy and Inflammation in a Rodent Model of Glaucoma
}

\author{
Wendi S. Lambert ${ }^{1}$, Brian J. Carlson ${ }^{1}$, Cathryn R. Formichella ${ }^{1}$, Rebecca M. Sappington ${ }^{1}$, \\ Clarence Ahlem ${ }^{2}$ and David J. Calkins ${ }^{1 *}$ \\ ${ }^{1}$ Vanderbilt University Medical Center, The Vanderbilt Eye Institute, Nashville, TN, USA, ${ }^{2}$ NeurMedix, Inc., San Diego, CA, \\ USA
}

Glaucoma is a group of optic neuropathies associated with aging and sensitivity to intraocular pressure (IOP). The disease is the leading cause of irreversible blindness worldwide. Early progression in glaucoma involves dysfunction of retinal ganglion cell (RGC) axons, which comprise the optic nerve. Deficits in anterograde transport along RGC axons to central visual structures precede outright degeneration, and preventing

\section{OPEN ACCESS}

Edited by:

Jason R. Richardson,

Northeast Ohio Medical University,

USA

Reviewed by:

Adriana Di Polo,

University of Montreal, Canada

Mohammad Harun-Or-Rashid,

Northeast Ohio Medical University,

USA

*Correspondence:

David J. Calkins

david.j.calkins@vanderbilt.edu

Specialty section:

This article was submitted to

Neurodegeneration,

a section of the journal

Frontiers in Neuroscience

Received: 28 September 2016

Accepted: 20 January 2017

Published: 07 February 2017

Citation:

Lambert WS, Carlson BJ, Formichella CR, Sappington RM, Ahlem C and Calkins DJ (2017) Oral

Delivery of a Synthetic Sterol Reduces

Axonopathy and Inflammation in a

Rodent Model of Glaucoma.

Front. Neurosci. 11:45.

doi: 10.3389/fnins.2017.00045 these deficits is efficacious at abating subsequent progression. HE3286 is a synthetic sterol derivative that has shown therapeutic promise in models of inflammatory disease and neurodegenerative disease. We examined the efficacy of HE3286 oral delivery in preventing loss of anterograde transport in an inducible model of glaucoma (microbead occlusion). Adult rats received HE3286 (20 or $100 \mathrm{mg} / \mathrm{kg}$ ) or vehicle daily via oral gavage for 4 weeks. Microbead occlusion elevated IOP 30\% in all treatment groups, and elevation was not affected by HE3286 treatment. In the vehicle group, elevated IOP reduced anterograde axonal transport to the superior colliculus, the most distal site in the optic projection, by $43 \%(p=0.003)$; HE3286 (100 mg/kg) prevented this reduction $(p=0.025)$. HE3286 increased brain-derived neurotrophic factor (BDNF) in the optic nerve head and retina, while decreasing inflammatory and pathogenic proteins associated with elevated IOP compared to vehicle treatment. Treatment with HE3286 also increased nuclear localization of the transcription factor $\mathrm{NF}_{\kappa \mathrm{B}}$ in collicular and retinal neurons, but decreased NFKB in glial nuclei in the optic nerve head. Thus, HE3286 may have a neuroprotective influence in glaucoma, as well as other chronic neurodegenerations.

Keywords: glaucoma, neuroinflammation, neuroprotection, axonopathy, HE3286, NF kappa B, axonal transport, brain derived neurotrophic factor

\section{INTRODUCTION}

Glaucoma is the leading cause of irreversible blindness worldwide (Quigley and Broman, 2006). The disease selectively targets retinal ganglion cells (RGCs) and their axons through stress conveyed at the optic nerve head (Calkins, 2012; Nickells et al., 2012). Age is a major risk factor for developing glaucoma, but sensitivity to intraocular pressure (IOP) is the only modifiable risk factor and sole target for clinical intervention (Heijl et al., 2002). Decreasing IOP with drugs or surgery can slow 
disease progression, but for many patients does not prevent RGC degeneration and vision loss (Heijl et al., 2002). Given that by 2020 nearly 80 million people will have glaucoma, with an estimated 11.2 million with permanent vision loss (Quigley and Broman, 2006; Cheung et al., 2008; Schober et al., 2008), the identification of therapeutic agents that can protect RGCs independently of IOP is of paramount importance.

HE3286 (17 $\alpha$-Ethynyl-androst-5ene-3 $\beta, 7 \beta, 17 \beta$-triol) is a synthetic derivative of androst-5-ene- $3 \beta, 7 \beta, 17 \beta$-triol (AET), a non-glucocorticoid anti-inflammatory metabolite of the adrenal steroid, dehydroepiandrosterone (DHEA; Ahlem et al., 2011). DHEA and AET have shown therapeutic promise in rodent models of immune-mediated inflammatory disorders with varying results in clinical trials (Offner et al., 2002; Dillon, 2005; Ahlem et al., 2009). HE3286 shows better oral bioavailability in humans, has low potential for toxicity, does not bind to glucocorticoid receptor or any known nuclear hormone receptor, and is not immunosuppressive (Wang et al., 2010; Ahlem et al., 2011). Treatment with HE3286 improves outcome measures in models of autoimmune disease, lung inflammation, experimental optic neuritis and Parkinson's disease, and is currently being tested in clinical trials (Auci et al., 2007, 2010; Ahlem et al., 2009; Offner et al., 2009; Conrad et al., 2010; Lu et al., 2010; Kosiewicz et al., 2011; Nicoletti et al., 2012; Reading et al., 2013a,b; Khan et al., 2014). It is believed HE3286 may act via binding, regulation and/or activation of MAPK or ERK, or through modulation of NFKB (Lu et al., 2010; Ahlem et al., 2011; Nicoletti et al., 2012; Reading et al., 2012).

Neurodegeneration in glaucoma shows many similarities with other age-related neurodegenerative disorders, including early deficits in axon function that precede loss of neurons themselves (Crish and Calkins, 2011; Mckinnon, 2012; Ghiso et al., 2013; Jindal, 2013; Danesh-Meyer and Levin, 2015; Jain and Aref, 2015). In animal models of glaucoma, degradation of RGC anterograde transport to central brain targets occurs early and prior to outright loss of axons in the optic projection (Crish et al., 2010, 2013; Dengler-Crish et al., 2014). Experimental interventions that prevent transport deficits are efficacious at stopping subsequent degeneration of axons and cell bodies (Lambert et al., 2011; Dapper et al., 2013). Here, we tested whether HE3286 could rescue RGC axon transport and modulate markers for neuroinflammation in the optic projection using our inducible microbead occlusion model of glaucoma in rats (Crish et al., 2010; Sappington et al., 2010; Dapper et al., 2013). We found that while HE3286 had no effect on IOP, daily oral delivery for 4 weeks prevented deficits in anterograde transport to the superior colliculus (SC), the primary central projection for RGCs in rodents (Linden and Perry, 1983; Hofbauer and Drager, 1985). HE3286 also influenced the level of many proteins implicated in the pathogenesis of glaucoma and other neurodegenerative disorders throughout the optic projection. Finally, HE3286 increased activation of the transcription factor $\mathrm{NF \kappa B}$ in colliculus and retina, while decreasing glial $\mathrm{NF \kappa B}$ activation in the optic nerve head, which is a major pathogenic site of neuroinflammation in glaucoma (Soto and Howell, 2014; Russo et al., 2016). These data suggest HE3286 has therapeutic potential with regards to neurodegeneration in glaucoma by modulating common neuroinflammatory pathways.

\section{MATERIALS AND METHODS}

\section{Animals}

All experimental procedures were conducted in accordance with the guidelines of and approved by The Vanderbilt University Institutional Animal Care and Use Committee. Brown Norway rats (7-9 months old, male) were obtained from Charles River Laboratories (Wilmington, MA) and maintained in a 12-h lightdark cycle with standard rodent chow available ad libitum. Three cohorts of rats ( $n=6$ per cohort; 18 rats total) were randomly assigned to one of three treatment groups: vehicle, $20 \mathrm{mg} / \mathrm{kg}$ HE3286 or $100 \mathrm{mg} / \mathrm{kg}$ HE3286. We measured IOP bilaterally in awake rats using a TonoPen XL rebound tonometer (Medtronic Solan, Jacksonville, FL) as previously described (Sappington et al., 2010; Crish et al., 2013; Dapper et al., 2013). To avoid corneal irritation, hydrating eye drops were administered to each eye at the completion of IOP measurements. Prior to microbead occlusion (Sappington et al., 2010; Crish et al., 2013; Dapper et al., 2013), we monitored IOP for 2-3 days; these measurements were averaged to obtain a baseline value. We elevated IOP unilaterally (OS) by a single $5.0 \mu \mathrm{l}$ injection of $15 \mu \mathrm{m}$ polystyrene microbeads (Molecular Probes, Eugene, OR) into the anterior chamber. The fellow eye $(\mathrm{OD})$ received an equivalent volume of saline to serve as an internal control. Beginning $24 \mathrm{~h}$ post-injection (day 1), we monitored IOP using tonometry at least three times weekly for the duration of the experiment (Sappington et al., 2010; Crish et al., 2013; Dapper et al., 2013). Beginning with the microbead injection (day 0 ), rats received $20 \mathrm{mg} / \mathrm{kg}$ or $100 \mathrm{mg} / \mathrm{kg}$ HE3286 $(10 \mathrm{mg} / \mathrm{mL}$ HE3286 in an aqueous medium containing $1 \mathrm{mg} / \mathrm{mL}$ sodium carboxymethyl cellulose, $9 \mathrm{mg} / \mathrm{mL}$ sodium chloride, $20 \mathrm{mg} / \mathrm{mL}$ polysorbate- 80 , and $0.5 \mathrm{mg} / \mathrm{mL}$ phenol as abroad spectrum preservative, Harbor Therapeutics, San Diego, CA 92122) via oral gavage. For the vehicle group, half received $20 \mathrm{mg} / \mathrm{kg}$ vehicle and the other half $100 \mathrm{mg} / \mathrm{kg}$ vehicle $(1 \mathrm{mg} / \mathrm{mL}$ sodium carboxymethyl cellulose, $9 \mathrm{mg} / \mathrm{mL}$ sodium chloride, 20 $\mathrm{mg} / \mathrm{mL}$ polysorbate- 80 , and $0.5 \mathrm{mg} / \mathrm{mL}$ phenol in an aqueous medium, Harbor Therapeutics, San Diego, CA 92122). Rats received vehicle or HE3286 once daily via oral gavage for 28 days.

\section{Anterograde Axonal Transport}

Forty-eight hours prior to perfusion, rats were anesthetized with $2.5 \%$ isoflurane and injected intravitreally with $2 \mu \mathrm{l}$ of $0.5 \mathrm{mg}$ cholera toxin subunit B (CTB) conjugated to Alexa Fluor-488 (Molecular Probes, CA) as previously described (Crish et al., 2010; Dapper et al., 2013; Ward et al., 2014). Animals were transcardially perfused with phosphate buffered saline (PBS) followed with $4 \%$ paraformaldehyde in PBS. Brains were cryoprotected overnight in 30\% sucrose/PBS and coronal midbrain sections $(50 \mu \mathrm{m})$ cut on a freezing sliding microtome. Alternating sections of superior colliculus (SC) were imaged using a Nikon Ti Eclipse microscope (Nikon Instruments Inc., Melville, NY) and the intensity of CTB signal was quantified using a custom ImagePro macro (Media Cybernetics, Bethesda, MD) as previously described (Crish et al., 2010; Dapper et al., 
2013; Ward et al., 2014). After normalizing to background, CTB signal intensity was calculated to reconstruct a retinotopic map of intact anterograde transport across the SC. Percent of intact transport for each map was defined as the region of the SC with intensity $\geq 70 \%$ of the maximum CTB signal for that tissue. CTB uptake by RGCs in the retina was verified using a Zeiss FV-1000 inverted confocal microscope through the Vanderbilt University Medical Center Cell Imaging Shared Resource.

\section{Immunohistochemistry}

Whole eyes were dissected from perfused animals, paraffin-embedded and vertically sectioned (6 $\mu \mathrm{m})$. Immunohistochemistry of whole eyes and brain was performed as previously described and at identical conditions between cohorts (Sappington et al., 2009; Crish et al., 2010; Weitlauf et al., 2014). Primary antibodies used to immunolabel proteins of interest in the brain, optic nerve head, and retina are listed in Table 1. Sections were incubated with appropriate secondary antibodies (1:200; Jackson ImmunoResearch Laboratories, Inc., West Grove, PA) and then cover-slipped with DAPI Fluoromount G (Southern Biotech, Birmingham, AL). Sections were imaged using a Zeiss FV-1000 inverted confocal microscope through the Vanderbilt University Medical Center Cell Imaging Shared Resource. For retina, all images were collected within the mid-peripheral region for comparison across eyes and cohorts. Identical microscope settings were used to acquire images for signal quantification, which was performed by a

TABLE 1 | Primary antibodies used for brain and whole eye immunohistochemistry.

\begin{tabular}{|c|c|c|c|}
\hline Protein & Dilution used & Catalog number & Vendor \\
\hline Alzheimer precursor protein (APP) & $1: 50$ & MAB348 & 1 \\
\hline$\beta$-amyloid $(A \beta)$ & $1: 200$ & \#2454 & 2 \\
\hline $\begin{array}{l}\text { Brain derived neurotrophic factor } \\
\text { (BDNF) }\end{array}$ & $1: 100$ & NBP2-42215 & 3 \\
\hline CD44 & $1: 150$ & NB600-1317 & 3 \\
\hline C1q & $1: 1000$ & A301 & 4 \\
\hline Ceruloplasmin (Cp) & $1: 500$ & \#611488 & 5 \\
\hline Choline acetyltransferase (ChAT) & $1: 100$ & AB144P & 1 \\
\hline Glial fibrillary acidic protein (GFAP) & $1: 500$ & MAB360 & 1 \\
\hline Interleukin $1 \beta$ (IL1 $\beta$ ) & $1: 50$ & AF-501-NA & 6 \\
\hline Interleukin 6 (IL6) & $1: 400$ & ab6672 & 7 \\
\hline IL6R $\alpha$ membrane bound (IL6R $\alpha$ m) & $1: 50$ & sc-600 & 8 \\
\hline $\begin{array}{l}\text { Ionized } \mathrm{Ca}^{2+} \text {-binding adapter } \\
\text { molecule } 1 \text { (lba1) }\end{array}$ & $1: 400$ & ab107159 & 7 \\
\hline NeuN & $1: 500$ & NBP1-92693 & 3 \\
\hline NF-кB & $1: 100$ & sc-372 & 8 \\
\hline p75 neurotrophin receptor (p75) & $1: 500$ & G323A & 9 \\
\hline $\begin{array}{l}\text { Phosphorylated neurofilament-H } \\
(\mathrm{pNF}-\mathrm{H})\end{array}$ & $1: 1000$ & 801601 & 10 \\
\hline Tumor necrosis factor $\alpha(\mathrm{TNF} \alpha)$ & $1: 300$ & AF-510-NA & 6 \\
\hline
\end{tabular}

1, EMD Millipore, Billerica, MA; 2, Cell Signaling Technology, Danvers, MA; 3, Novus Biologicals, Littleton CO; 4, Quidel Corp., San Diego, CA; 5, BD Transductions Labs, San Jose, CA; 6, R and D Systems, Minneapolis, MN; 7, AbCam, Cambridge, MA; 8, Santa Cruz Biotechnologies, Dallas, TX; 9, Promega Corp., Madison, WI; 10, BioLegend, San Diego, CA. naïve observer using a custom macro in ImagePro (Media Cybernetics; Bethesda, MD) that determines the percent area of the positive label (Crish et al., 2013). The microbead to saline ratio (microbead:saline) of label in a specific tissue was calculated for each animal; ratios were averaged and reported as label (microbead:saline). Nuclear localization of NFkB was analyzed using a custom macro in ImageJ (Carmona et al., 2007; Schneider et al., 2012) followed by signal quantification in ImagePro. For quantifying label, a tissue section serving each saline and microbead eye from at least five animals per cohort was used. Retinal and nuclear layer thickness were measured at five different retinal locations per image using the Measurement tool in ImagePro. For RGC counts, the number of CTB-positive and DAPI-positive cells within the ganglion cell layer were counted using the Count tool in Photoshop. At least eight images per animal were quantified to determine an average retinal thickness.

\section{Statistical Analysis}

All data are expressed as mean \pm standard error unless indicated otherwise. The number of samples used in each experiment is provided in the appropriate methods description or figure legend. Statistical comparisons between two independent measurements were made using two-sided $t$-tests, following confirmation of normality for each using the Shapiro-Wilk normality test; samples for which normality failed were compared using the Mann-Whitney Rank Sum Test (SigmaPlot 12.5, Systat Software, Inc., Chicago, IL). Comparisons between multiple groups were made using one way analysis of variance (ANOVA) followed by the Holm-Sidak Pairwise Multiple Comparison test (SigmaPlot 11.1, Systat Software, Inc., Chicago, IL). Comparisons of a sample mean to a hypothesized or predicted value were made using a one-sided $t$-test. Actual $p$-values of significance are indicated where appropriate in the results section or figure legends. All comparisons for which significance is reported achieved or exceeded a post-hoc calculation of power of 0.80 .

\section{RESULTS}

Two rats, one from each HE3286 group, were euthanized before completion of the study due to complications from oral gavage. Treatment with HE3286 for 28 days had few directly observable adverse effects, as rats were active, responsive and showed no overt signs of distress. HE3286 did result in weight loss in both the 20 and $100 \mathrm{mg} / \mathrm{kg}$ groups (Table 2). Weights on day 0 (first day of treatment) ranged from 283 to $430 \mathrm{~g}$, and mean

TABLE 2 | Weight loss with HE3286 treatment following microbead
injection.
\begin{tabular}{lcccc}
\hline Treatment group & Weight day 0 (g) & Weight day 28 (g) & $\%$ Change (\%) \\
\hline Vehicle & $357.2 \pm 19.7$ & $371.5 \pm 21.0$ & +4.03 \\
20 mg/kg HE3286 & $347.5 \pm 6.5$ & $326.3 \pm 9.8$ & $-6.16^{*}$ \\
100 mg/kg HE3286 & $371.7 \pm 15.0$ & $333.7 \pm 14.7$ & $-10.26^{* \dagger}$ \\
\hline
\end{tabular}

${ }^{*} p<0.001$ compared to vehicle; ${ }^{\dagger} p=0.042$ compared to $20 \mathrm{mg} / \mathrm{kg}$ dose $(n=6$ rats per group). 
weights for the three treatment groups were not significantly different $(p=0.523)$. Weights on day 28 (last day of treatment) ranged from 290 to $440 \mathrm{~g}$, with no significant difference in mean weight between the three treatment groups $(p=0.130)$. However, rats that received vehicle gained $14.3 \mathrm{~g}$ during the experimental period, while rats that received $20 \mathrm{mg} / \mathrm{kg}$ HE3286 lost $21.2 \mathrm{~g}$ and rats that received $100 \mathrm{mg} / \mathrm{kg}$ HE3286 lost $38.0 \mathrm{~g}$. Comparing percent change in weight over the course of treatment for each animal showed that HE3286 decreased weight in both the 20 $\mathrm{mg} / \mathrm{kg}$ group ( $p<0.001$, compared to vehicle) and $100 \mathrm{mg} / \mathrm{kg}$ group ( $p<0.001$, compared to vehicle; $p=0.042$ compared to 20 $\mathrm{mg} / \mathrm{kg})$.

\section{Oral Delivery of HE3286 Has no Effect on Ocular Pressure}

Baseline IOP measurements ranged from $19.15 \pm 0.50$ to 20.29 $\pm 0.55 \mathrm{~mm} \mathrm{Hg}$ and were similar for all treatment groups $(p=$ 0.638). Microbead injection elevated IOP in all eyes for the full 28 day period regardless of treatment (Figure 1A). In vehicletreated rats, elevation was $32.7 \%$ compared to saline-injected eyes: $26.69 \pm 2.73$ vs. $20.12 \pm 2.69 \mathrm{~mm} \mathrm{Hg} ; p=0.002$ (Figure 1B). Similar increases of $29.6 \%$ and $30.0 \%$ were observed for the 20 $\mathrm{mg} / \mathrm{kg}$ HE3286-treated group $(26.39 \pm 2.51$ vs. $20.37 \pm 2.68 \mathrm{~mm}$ $\mathrm{Hg}, p=0.002)$ and for the $100 \mathrm{mg} / \mathrm{kg}$ HE3286-treated group $(26.49 \pm 2.52$ vs. $20.39 \pm 2.53 \mathrm{~mm} \mathrm{Hg}, p=0.002)$, respectively. Mean IOP of saline eyes did not significantly differ for any treatment group ( $p=0.997)$; the same was true for microbead eyes $(p=0.997)$.

\section{HE3286 Rescues Anterograde Axonal Transport to the Superior Colliculus}

Deficits in anterograde axonal transport from retina to SC are an early sign of pathogenesis in rodent models of glaucoma, including microbead occlusion (Crish et al., 2010; Lambert et al., 2011; Dapper et al., 2013; Ward et al., 2014). Figure 2 compares the level of intact transport of fluorescently labeled CTB to the SC of vehicle-, $20 \mathrm{mg} / \mathrm{kg} \mathrm{HE3286-,} \mathrm{and} 100 \mathrm{mg} / \mathrm{kg}$ HE3286-treated rats. Deficits in transport are readily apparent in the SC from microbead-injected eyes of vehicle rats (dotted lines, Figure 2A top row). These deficits tend to fill in complete retinotopic sectors when reconstructed from serial sections through the SC (Figure 2A bottom row), consistent with our previous studies (Lambert et al., 2011; Dapper et al., 2013). Variability in transport was low in SC from saline-injected eyes (Figure 2B), which had similarly intact transport for all groups: $89.2 \pm 2.7 \%$ for vehicle, $85.8 \pm 3.9 \%$ for $20 \mathrm{mg} / \mathrm{kg} \mathrm{HE} 3286$, and $90.9 \pm 3.7 \%$ for $100 \mathrm{mg} / \mathrm{kg}$ HE3286 (Figure 2C; $p=0.593$ ). Elevated IOP due to microbead injection decreased intact transport to the SC in vehicle-treated rats to $57.4 \pm 6.0 \%$ intact transport (Figure 2C; $p$ $=0.003$ compared to saline). Treatment with HE3286 attenuated transport deficits dramatically, as intact transport to the SC from microbead-injected eyes was $71.9 \pm 9.8$ and $83.0 \pm 7.1 \%$ for the 20 and $100 \mathrm{mg} / \mathrm{kg} \mathrm{HE} 3286$ treatment groups, respectively (Figure 2C); neither differed from intact transport from the saline eye ( $p \geq 0.270)$. Compared to SC from microbead-injected eyes in the vehicle group, the improvement was significant for the $100 \mathrm{mg} / \mathrm{kg} \mathrm{HE} 3286$ cohort $(p=0.025)$. No difference in SC volume was detected for any groups or any treatments $(p=$ $0.082)$.

\section{HE3286 Counters the Influence of Elevated IOP Throughout the Optic Projection}

\section{Brain-Derived Neurotrophic Factor (BDNF)}

Deficits in anterograde transport to the SC of glaucomatous mice is accompanied by significant changes in local neurochemistry, including increased localization of BDNF (Crish et al., 2013). Within the SC of vehicle rats, BDNF also appeared to increase with microbead-induced elevated IOP (Figure 3A, left); treatment with $\mathrm{HE} 3286(20 \mathrm{mg} / \mathrm{kg})$ appeared to blunt this increase (Figure 3A, right). In the optic nerve head $(\mathrm{ONH}$; Figure 3B), which is a major site of stress in glaucoma (Hernandez, 2000), levels of BDNF appeared similar in salineand microbead-injected eyes from vehicle treated rats. Treatment with HE3286 $(20 \mathrm{mg} / \mathrm{kg})$ appeared to increase BDNF in the $\mathrm{ONH}$ of microbead-injected eyes compared to saline. Finally, levels of BDNF in the retina decreased following microbead injection in vehicle-treated rats, with HE3286 again blunting this effect (Figure 3C).

When quantified (Figure 3D), the ratio of BDNF in microbead to saline SC for vehicle rats was significantly greater than an expected ratio of unity: $2.34 \pm 0.12(p<0.001)$. This ratio was diminished by $70 \%$ compared to vehicle in the $20 \mathrm{mg} / \mathrm{kg}$ HE3286 group $(0.71 \pm 0.09 ; p<0.001)$ and by $43 \%$ in the 100 $\mathrm{mg} / \mathrm{kg}$ group $(1.34 \pm 0.08 ; p<0.001)$. In the $\mathrm{ONH}$ (Figure 3D, middle), the ratio of microbead to saline BDNF increased with $20 \mathrm{mg} / \mathrm{kg}$ HE3286 by $69 \%: 1.43 \pm 0.20$ vs. $0.84 \pm 0.16$ for vehicle $(p=0.042)$. The $34 \%$ increase in this ratio for the $100 \mathrm{mg} / \mathrm{kg}$ dose was not significant compared to vehicle $(0.93 \pm 0.12 ; p=$ 0.66). Finally, in the retina, treatment with HE3286 increased the microbead:saline ratio of BDNF by $282 \%$ in the $20 \mathrm{mg} / \mathrm{kg}$ group $(0.64 \pm 0.05)$ and by $501 \%$ in the $100 \mathrm{mg} / \mathrm{kg}$ group $(1.00$ $\pm 0.05)$ when compared to vehicle $(0.17 \pm 0.04 ; p<0.002)$; the $100 \mathrm{mg} / \mathrm{kg} \mathrm{HE} 3286$ ratio was significantly below an expected ratio of unity $(p=0.02)$. Thus, as microbead-induced elevations in IOP degrade anterograde transport in the optic projection, HE3286 treatment generally has the effect of opposing the change in BDNF levels induced by elevated IOP.

Note that for BDNF and all other immunolabeled proteins we quantified, the total SC area for saline $\left(7.013 \pm 0.006 \mathrm{~mm}^{2}\right)$ and microbead $\left(7.017 \pm 0.003 \mathrm{~mm}^{2}\right)$ eyes or vehicle $(7.019 \pm 0.002$ $\left.\mathrm{mm}^{2}\right)$ and HE3286 groups $\left(7.012 \pm 0.005 \mathrm{~mm}^{2}\right)$ did not vary $(p \geq 0.088)$. Similarly, total area quantified in the nerve head did not vary between saline $\left(7.019 \pm 0.002 \mathrm{~mm}^{2}\right)$ and microbead $\left(7.022 \pm 0.002 \mathrm{~mm}^{2}\right)$ eyes or between vehicle $(7.018 \pm 0.003$ $\mathrm{mm}^{2}$ ) and HE3286 groups (7.022 $\left.\pm 0.002 \mathrm{~mm}^{2} ; p>0.279\right)$. Finally, total area quantified in the retina was similar between saline $\left(2.756 \pm 0.203 \mathrm{~mm}^{2}\right)$ and microbead $\left(2.712 \pm 0.198 \mathrm{~mm}^{2}\right)$ eyes or between vehicle $\left(2.996 \pm 0.204 \mathrm{~mm}^{2}\right)$ and HE3286 (2.603 $\left.\pm 0.199 \mathrm{~mm}^{2} ; p>0.076\right)$ groups.

\section{Microglia Activation}

Given HE3286's proposed anti-inflammatory action and the contribution of microglia as neuroinflammatory mediators in 

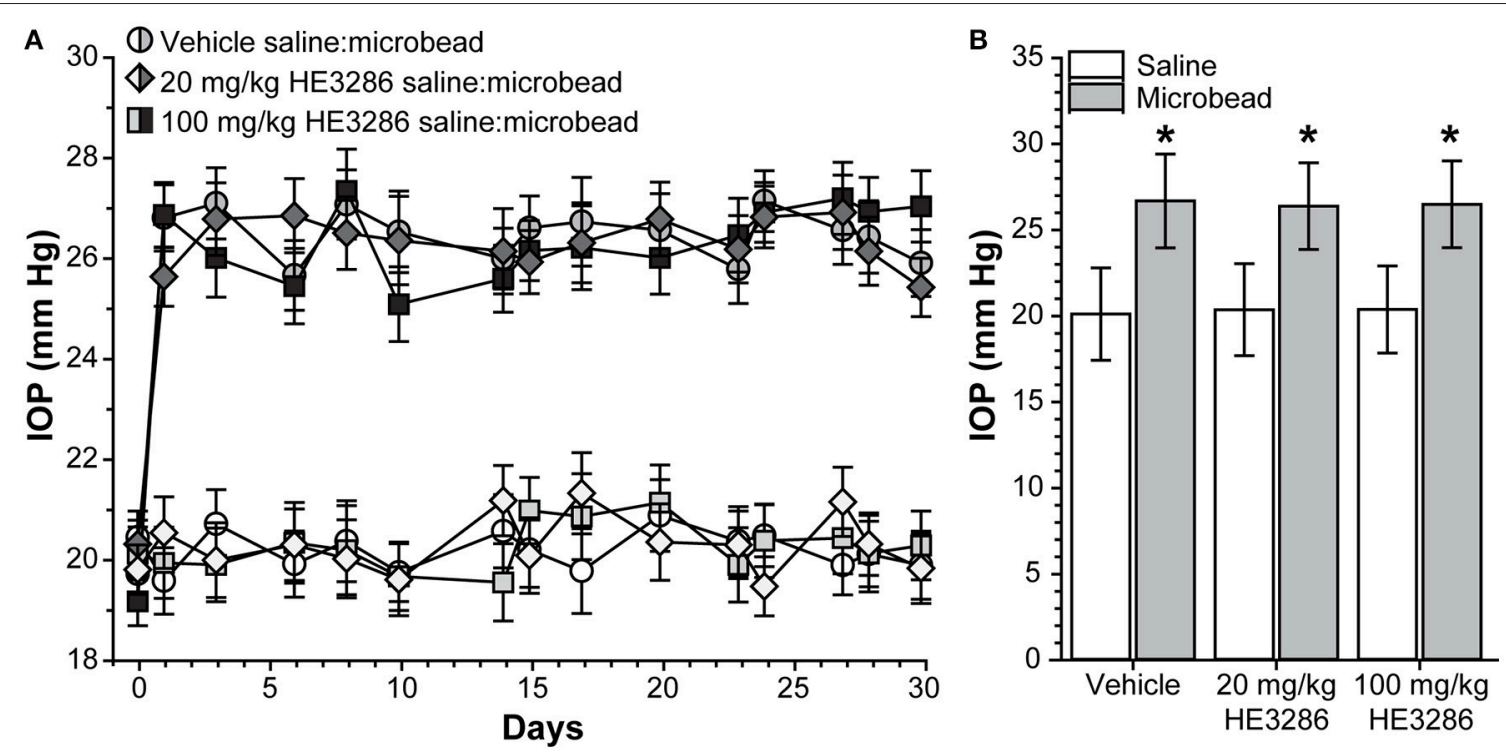

FIGURE 1 | HE3286 treatment does not lower ocular pressure following microbead occlusion. (A) Mean intraocular pressure (IOP) in rats before (day 0) and following (days $\geq 1$ ) a single unilateral injection of polystyrene microbeads $(5.0 \mu l)$ into the anterior chamber. The fellow eye was injected with an equivalent volume saline. (B) Microbead-injected eyes exhibited an increase in IOP following injection (mean \pm SEM shown) compared to saline eyes, regardless of treatment. ${ }^{\star} p \leq$ $0.002, n=6$ per treatment group.

glaucoma, we examined how treatment influences levels of ionized calcium-binding adapter molecule 1 (Iba1), a microgliaspecific marker (Ito et al., 1998; Soto and Howell, 2014; Mac Nair and Nickells, 2015). In the SC, microbead-induced elevated IOP elicited a modest increase in Iba1 levels in vehicle animals, which was mitigated by HE3286 (Figure 4A). In the ONH, elevated IOP sharply increased Ibal, which again was prevented in the HE3286 group (Figure 4B). Iba1 appeared elevated in microbead retina from vehicle-treated rats, while treatment with HE3286 had the opposite effect (Figure 4C). The influence of HE3286 was significant in all three tissues (Figure 4D). Treatment with the 20 $\mathrm{mg} / \mathrm{kg}$ dose of HE3286 reduced the ratio of Iba1 in microbead to saline SC by $63 \%$ : $0.84 \pm 0.11$ compared to $1.29 \pm 0.19$ for vehicle $(p=0.037)$. The $100 \mathrm{mg} / \mathrm{kg}$ dose had a similar influence $(0.86 \pm 0.17, p=0.061)$. The microbead:saline ratio of Ibal in $\mathrm{ONH}$ for vehicle rats was significantly greater than an expected ratio of unity: $1.56 \pm 0.12(p=0.045)$. This ratio was diminished by $83 \%$ in the $20 \mathrm{mg} / \mathrm{kg} \mathrm{HE} 3286$ group $(0.27 \pm 0.03 ; p=0.005)$; $100 \mathrm{mg} / \mathrm{kg} \mathrm{HE} 3286 \mathrm{had}$ far less effect $(1.09 \pm 0.27 ; p=0.193)$. For vehicle retina, the Ibal microbead to saline ratio was significantly more than $1(1.42 \pm 0.04 ; p=0.007)$. Treatment with both 20 $\mathrm{mg} / \mathrm{kg}$ and $100 \mathrm{mg} / \mathrm{kg}$ HE3286 decreased this ratio compared to vehicle: $1.09 \pm 0.08$ for the $20 \mathrm{mg} / \mathrm{kg}$ group $(p=0.018)$ and 0.99 $\pm 0.07(p=0.009)$ for the $100 \mathrm{mg} / \mathrm{kg}$ group.

\section{Markers of Neuroinflammation or Neurodegeneration}

Other significant markers implicated in neuroinflammation or neurodegeneration were also modulated in the optic projection by HE3286. Interleukin 6 (IL6) is a pro-inflammatory cytokine that can promote RGC survival (Matousek et al., 2012; Song et al., 2013). In the SC of vehicle rats, IL6 localization appeared to diminish with elevated IOP; this was reversed with HE3286 (Figure 5A). The p75 neurotrophin receptor is a member of the tumor necrosis factor receptor family and has diverse roles in neuronal activity, plasticity, and injury response (Meeker and Williams, 2015). We found that p75 levels increased within the $\mathrm{ONH}$ of microbead eyes from vehicle rats; treatment with $20 \mathrm{mg} / \mathrm{kg} \mathrm{HE} 3286$ prevented this increase (Figure 5B). Finally, amyloid precursor protein (APP) is cleaved to form $\beta$-amyloid, a major component of amyloid plaques. Recent evidence suggests this protein and its cleaved products may be involved in the pathogenesis of glaucoma (Jain and Aref, 2015). HE3286 appeared to increase APP levels in saline-injected eyes (Figure 5D). We found that microbead-induced elevations in IOP increased APP in the optic nerve head (Figure 5C) and retina (Figure 5D) of vehicle rats. In both tissues, HE3286 prevented this increase.

When quantified (Figure 5E), the ratio of IL6 in microbead to saline SC was significantly less than an expected value of unity: $0.55 \pm 0.08(p=0.005)$. This trend was reversed by 100 $\mathrm{mg} / \mathrm{kg}$ HE3286, which increased the IL6 ratio by $219 \%$ compared to vehicle: $1.75 \pm 0.51(p=0.047)$. In the ONH (Figure 5F), the microbead to saline ratio for $\mathrm{p} 75$ was also significantly higher than unity: $2.41 \pm 0.15(p=0.011)$. Treatment with both $20 \mathrm{mg} / \mathrm{kg}$ and $100 \mathrm{mg} / \mathrm{kg}$ HE3286 prevented the increase with elevated IOP, reducing the ratio by $58 \%(1.02 \pm 0.14 ; p=$ $0.002)$ and $66 \%(0.82 \pm 0.14 ; p=0.001)$, respectively, compared to vehicle. For APP in ONH (Figure 5G), treatment with 20 $\mathrm{mg} / \mathrm{kg}$ HE3286 decreased the microbead to saline ratio by $53 \%$ compared to vehicle: $5.71 \pm 1.15$ vs. $12.13 \pm 1.49(p=0.027)$. Similarly, $100 \mathrm{mg} / \mathrm{kg}$ HE3286 elicited a $54 \%$ decrease to 5.63 $\pm 0.84(p=0.019)$. In the retina (Figure 5H), elevated IOP 

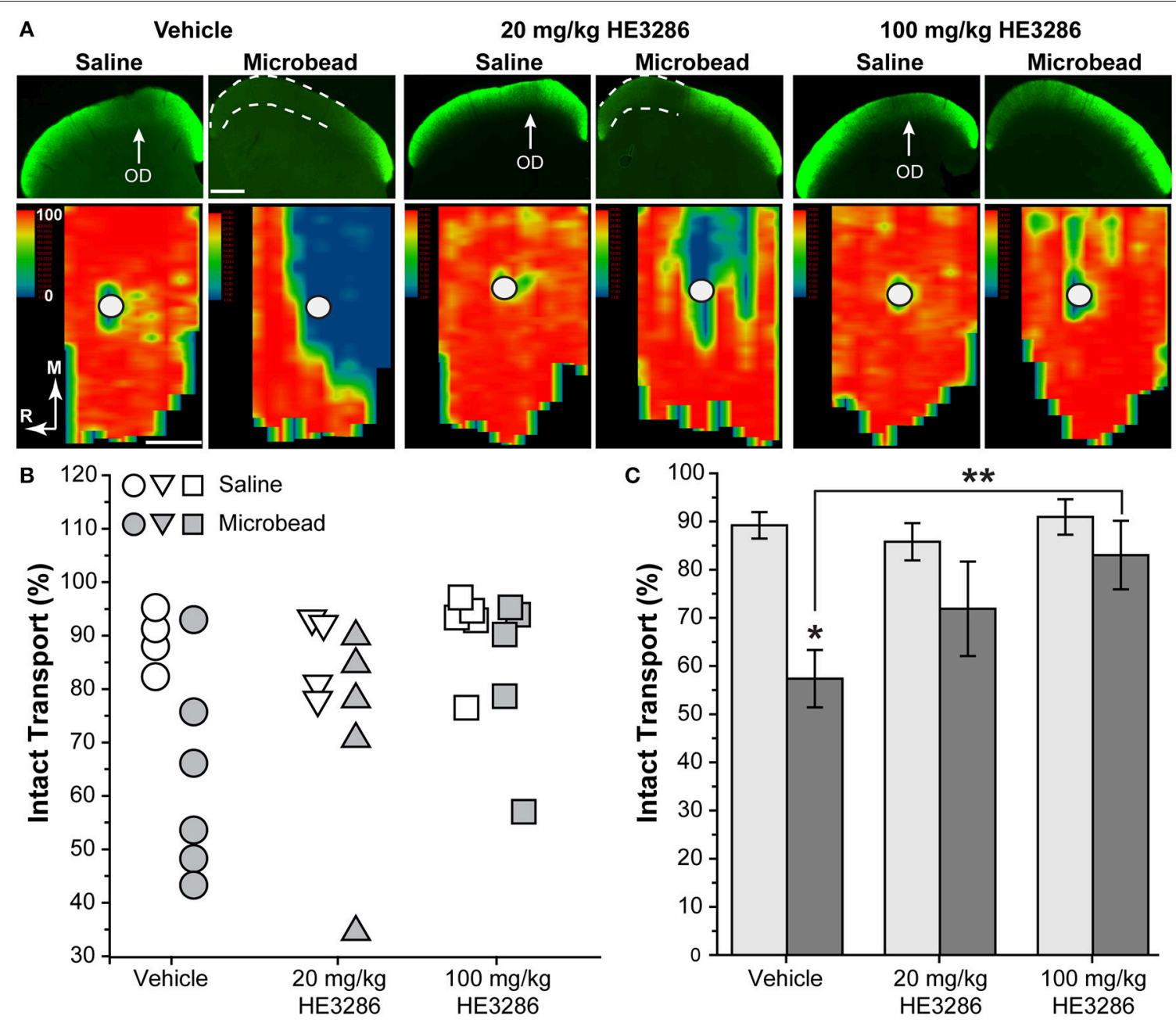

FIGURE 2 | HE3286 treatment rescues axonal transport following microbead occlusion. (A) Coronal sections (top row) through the superior colliculus following intravitreal injection of CTB (green) into saline- and microbead-injected eyes of vehicle- and HE3286-treated rats. Deficits in anterograde transport of CTB (dotted lines) due to microbead-induced elevations in IOP are most apparent in vehicle rats. Representation of the optic disc in the retina (OD) lacks transport due to absence of RGCs. Retinotopic maps (bottom row) reconstructed from serial sections of SC with optic disc indicated (circles). Density of the transported CTB signal ranges from 0\% (blue) to 50\% (green) to 100\% (red). Medial (M) and rostral (R) orientations are indicated. Scale: $500 \mu \mathrm{m}$. (B) Intact transport for individual saline- and microbead-injected eyes per treatment group given as fraction of SC retinotopic map with CTB signal $\geq 70 \%$ of the maximum. Two SCs from saline-injected eyes of vehicle-treated rats and one SC from a saline-injected 20 mg/kg HE3286-treated rat were excluded from analysis due to lack of CTB uptake by RGC. (C) Mean level of intact transport in SC was reduced by elevated IOP in vehicle group compared to saline eye $\left({ }^{*} p=0.003\right)$. Higher dose of HE3286 prevented this reduction $\left({ }^{\star *} p=\right.$ 0.025). $n=4-6$ animals per treatment group.

significantly increased the microbead to saline ratio for APP well above an expected ratio of unity: $8.05 \pm 0.81(p=0.013)$. Treatment with HE3286 significantly decreased the ratio by 97 and $94 \%$ for the $20 \mathrm{mg} / \mathrm{kg}$ and $100 \mathrm{mg} / \mathrm{kg}$ doses, respectively, compared to vehicle $(p<0.001)$. Both the ratio for $20 \mathrm{mg} / \mathrm{kg}$ HE3286 (0.25 \pm 0.05$)$ and $100 \mathrm{mg} / \mathrm{kg}$ HE3286 (0.48 \pm 0.14$)$ were significantly lower than unity $(p \leq 0.035)$.

As with BDNF (Figure 3) and Ibal (Figure 4), in each of the cases illustrated in Figure 5, treatment with one or both doses of HE3286 tended to oppose the action of elevated IOP in the vehicle cohort, as indicated by the directionality of the change in the microbead:saline ratio for each. If the ratio $>1$ in vehicle tissue, HE3286 treatment pushed the ratio below unity; if the vehicle ratio $<1$, HE3286 increased the ratio above unity. This tendency held for other proteins we tested in SC, ONH, and retina and for which HE3286 elicited a significant change in expression level. These results are summarized in Table 3.

\section{HE3286 Modulates Common Pathogenic Markers for Glaucoma in the Retina}

Next we examined how HE3286 treatment influences localization of common markers for neurodegeneration in glaucomatous retina. Ceruloplasmin $(\mathrm{Cp})$ is a positive acute phase protein upregulated during inflammation (Denko, 1979; Goldstein et al., 1979). In vehicle retina, Cp increased dramatically 

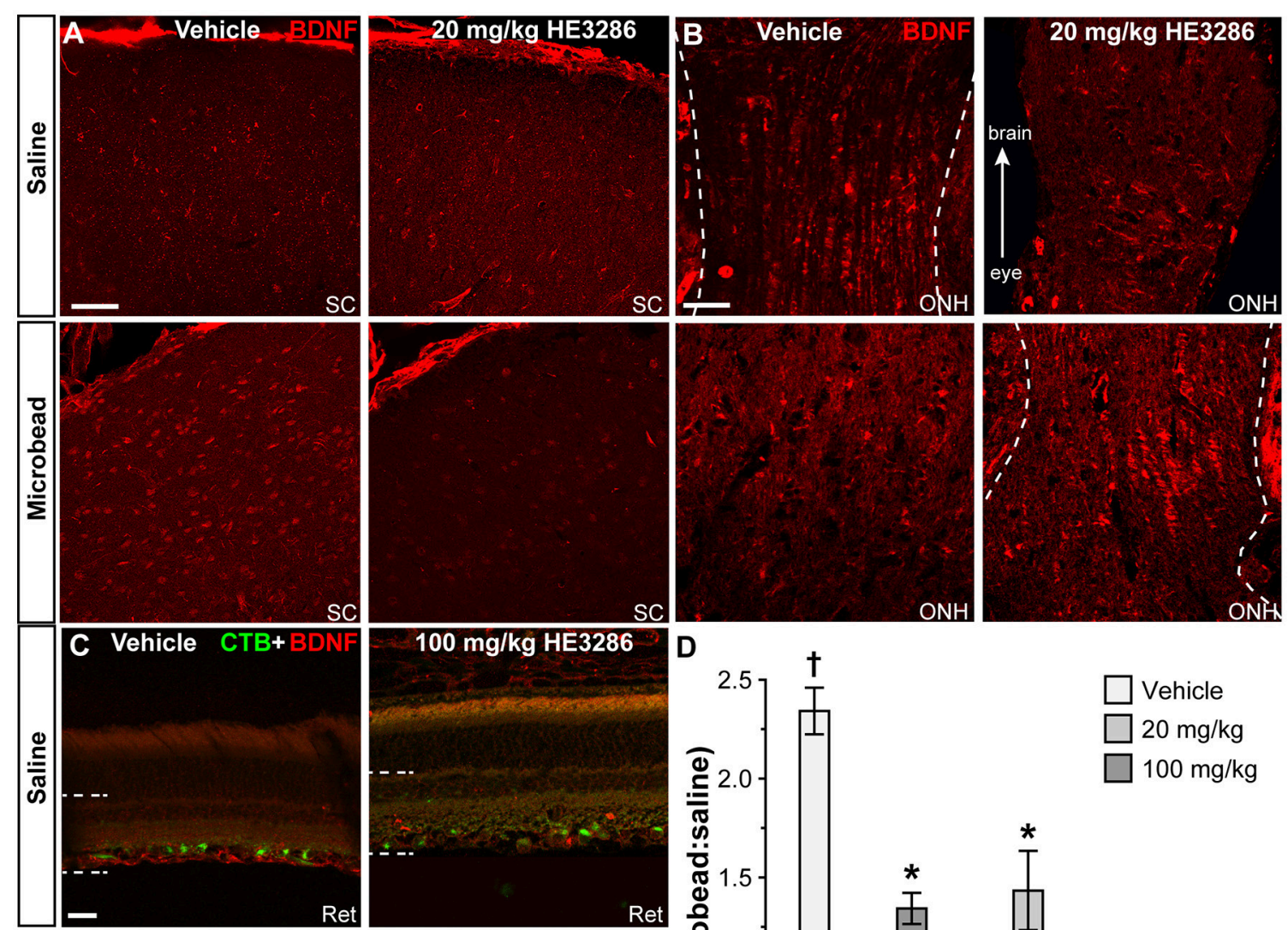

\section{D}

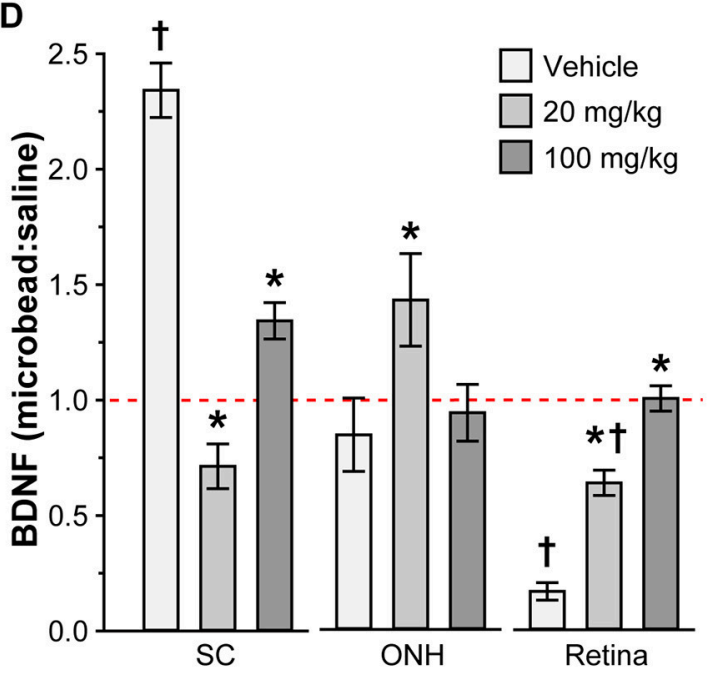

FIGURE 3 | HE3286 treatment influences BDNF levels in optic projection. Confocal micrographs of saline- and microbead-eye tissue from vehicle and HE3286 rats show immunolabeling for BDNF in superior colliculus (SC, A), optic nerve head (ONH, B), and retina (Ret, C), which also shows RGCs labeled by CTB uptake. Dotted lines indicate region of retina where immunolabel was quantified. Scale: $50 \mu \mathrm{m}$ (A,B) and $20 \mu \mathrm{m}$ (C). (D) Bar graphs indicate quantification of levels of BDNF in superior colliculus (SC), optic nerve head $(\mathrm{ONH})$, and retina expressed as the microbead:saline ratio for vehicle and $\mathrm{HE3286}$ treatment groups. ${ }^{\dagger}$ Indicates significant departure from expected ratio of unity $(p \leq 0.02)$, which is indicated (dotted line). *indicates $p \leq 0.042$ compared to ratio for vehicle group. $n=6$ animals per vehicle treatment group, 5 animals per $20 \mathrm{mg} / \mathrm{kg}$ and $100 \mathrm{mg} / \mathrm{kg}$ HE3286 groups for SC; $n=3$ animals per treatment group for ONH and retina analysis.

with microbead-induced elevated IOP; HE3286 prevented this increase (Figure 6A). Interleukin $1 \beta$ (IL1 $\beta$ ) is a proinflammatory cytokine that can promote neurodegeneration or protection depending on whether the injury is acute or chronic (Matousek et al., 2012; Song et al., 2013). In vehicle retina, IL1 $\beta$ decreased with elevated IOP (Figure 6B, left). While treatment with $20 \mathrm{mg} / \mathrm{kg} \mathrm{HE} 3286$ further reduced expression (images not shown), $100 \mathrm{mg} / \mathrm{kg}$ HE3286 reversed this trend (Figure 6B, right). In vehicle retina, elevated IOP appeared to increase expression of $\mathrm{Clq}$, an early component of the classical complement pathway, especially in CTB-labeled RGCs (Figure 6C, left). This too was prevented by $100 \mathrm{mg} / \mathrm{kg}$ HE3286
(Figure 6C, right). Finally, we also tested localization of choline acetyltransferase (ChAT), a marker for cholinergic amacrine cell neurons, to determine if HE3286 affects levels of common proteins not typically associated with glaucoma progression. Neither elevated IOP nor treatment appeared to influence localization levels (Figure 6D), which were consistent with other studies (Kang et al., 2004; Feng et al., 2006; Samuel et al., 2011).

When quantified, the ratio of $\mathrm{Cp}$ in microbead to saline retina in the vehicle group was well above an expected value of unity: $4.61 \pm 0.49$ ( $p=0.018$; Figure 6E). Treatment with HE3286 abated this increase by $80 \%$ compared to vehicle in the $20 \mathrm{mg} / \mathrm{kg}$ group $(0.92 \pm 0.29 ; p=0.003)$ and by $96 \%$ in the $100 \mathrm{mg} / \mathrm{kg}$ 

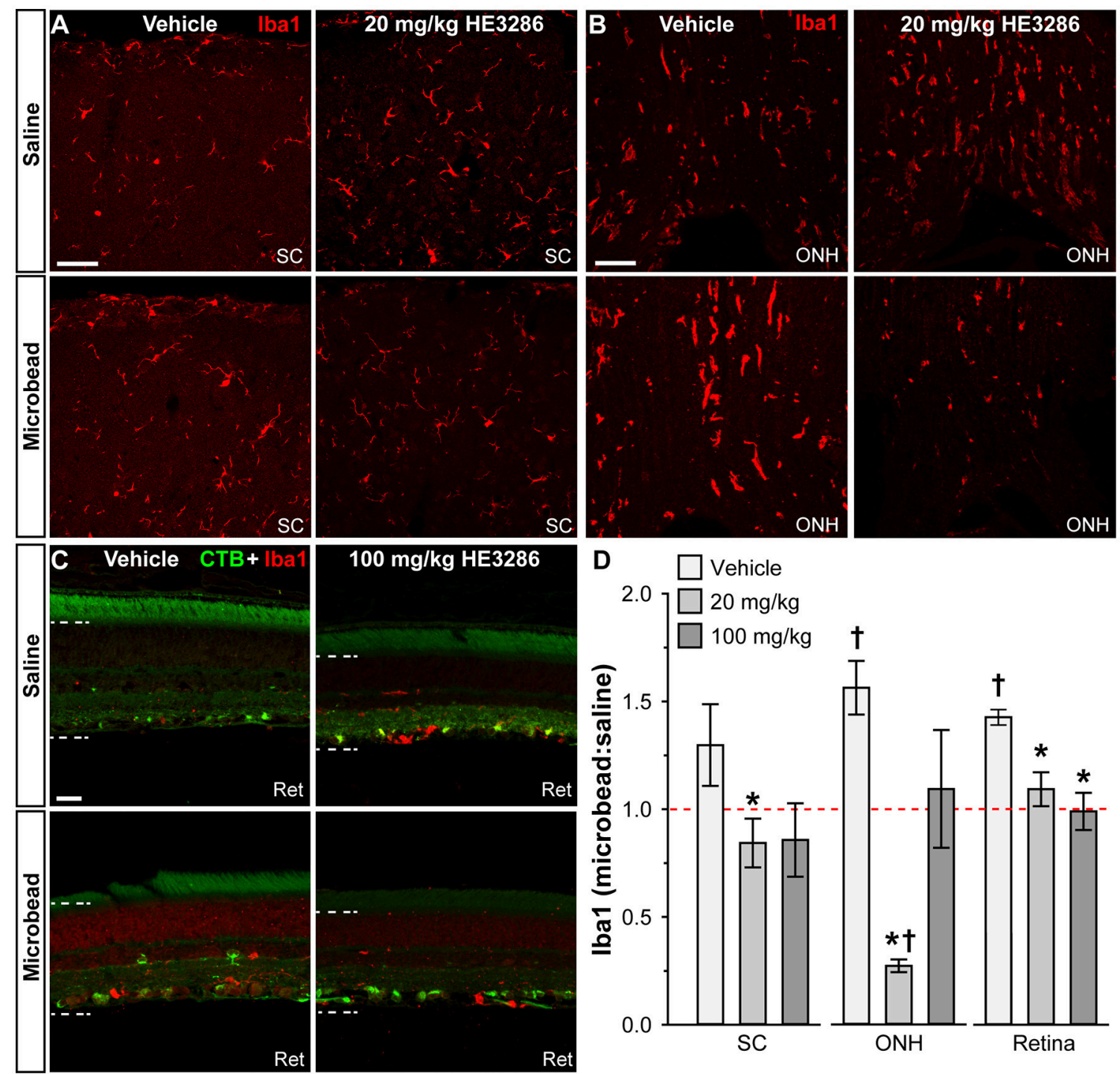

FIGURE 4 | HE3286 treatment influences lba1-labeled microglia in optic projection. Confocal micrographs of saline- and microbead-injected tissue from vehicle and HE3286 rats show immunolabeling for the microglia marker lba1 in superior colliculus (SC, A), optic nerve head (ONH, B), and retina (Ret, C), which also shows RGCs labeled by CTB uptake. Dotted lines indicate region of retina where immunolabel was quantified. Scale: $50 \mu \mathrm{m}$ (A,B) and $20 \mu \mathrm{m}$ (C). (D) Bar graphs indicate quantification of Iba1 in superior colliculus (SC), optic nerve head (ONH), and retina expressed as the microbead:saline ratio for vehicle and $\mathrm{HE} 3286$ treatment groups. ${ }^{\dagger}$ indicates significant departure from expected ratio of unity $(p \leq 0.045)$, which is indicated (dotted line). *Indicates $p \leq 0.037$ compared to ratio for vehicle group. $n=6$ animals per vehicle treatment group, 5 animals per $20 \mathrm{mg} / \mathrm{kg}$ and $100 \mathrm{mg} / \mathrm{kg}$ HE3286 groups for SC; $n=3$ animals per treatment group for ONH and retina analysis.

group $(0.18 \pm 0.03 ; p<0.001)$. For IL1 $\beta$ (Figure 6F), elevated IOP significantly reduced levels compared to saline retina in the vehicle group, yielding a ratio of $0.28 \pm 0.02(p=0.001)$. Treatment with $20 \mathrm{mg} / \mathrm{kg}$ HE3286 further reduced IL1 $\beta$ by $76 \%$ $(0.07 \pm 0.04 ; p=0.009)$. However, $100 \mathrm{mg} / \mathrm{kg}$ HE3286 had the opposite effect, increasing levels by $440 \%$ compared to vehicle $(1.49 \pm 0.14 ; p=0.001)$. Elevated IOP in vehicle rats significantly increased levels of $\mathrm{C1q}$ compared to saline retina: $1.225 \pm 0.061$ ( $p=0.033$, Figure 6G). While $20 \mathrm{mg} / \mathrm{kg} \mathrm{HE} 3286$ had little effect $(1.430 \pm 0.096), 100 \mathrm{mg} / \mathrm{kg}$ HE3286 significantly reduced the C1q microbead to saline ratio compared to vehicle: $0.037 \pm$
0.007 ( $p<0.001)$. As expected, levels of ChAT were the same in saline and microbead retinas in all treatment groups (Figure $\mathbf{6 H}$, $p \geq 0.461)$.

\section{HE3286 Changes NFKB Localization in the Optic Projection}

One proposed mechanism of action for HE3286 is to modulate the transcription factor $\mathrm{NF} \kappa \mathrm{B}$, which translocates to the nucleus when activated (Offner et al., 2009). Using an antibody with proven efficacy in brain (Herkenham et al., 2011; Figure 7A), we examined and quantified the degree of 

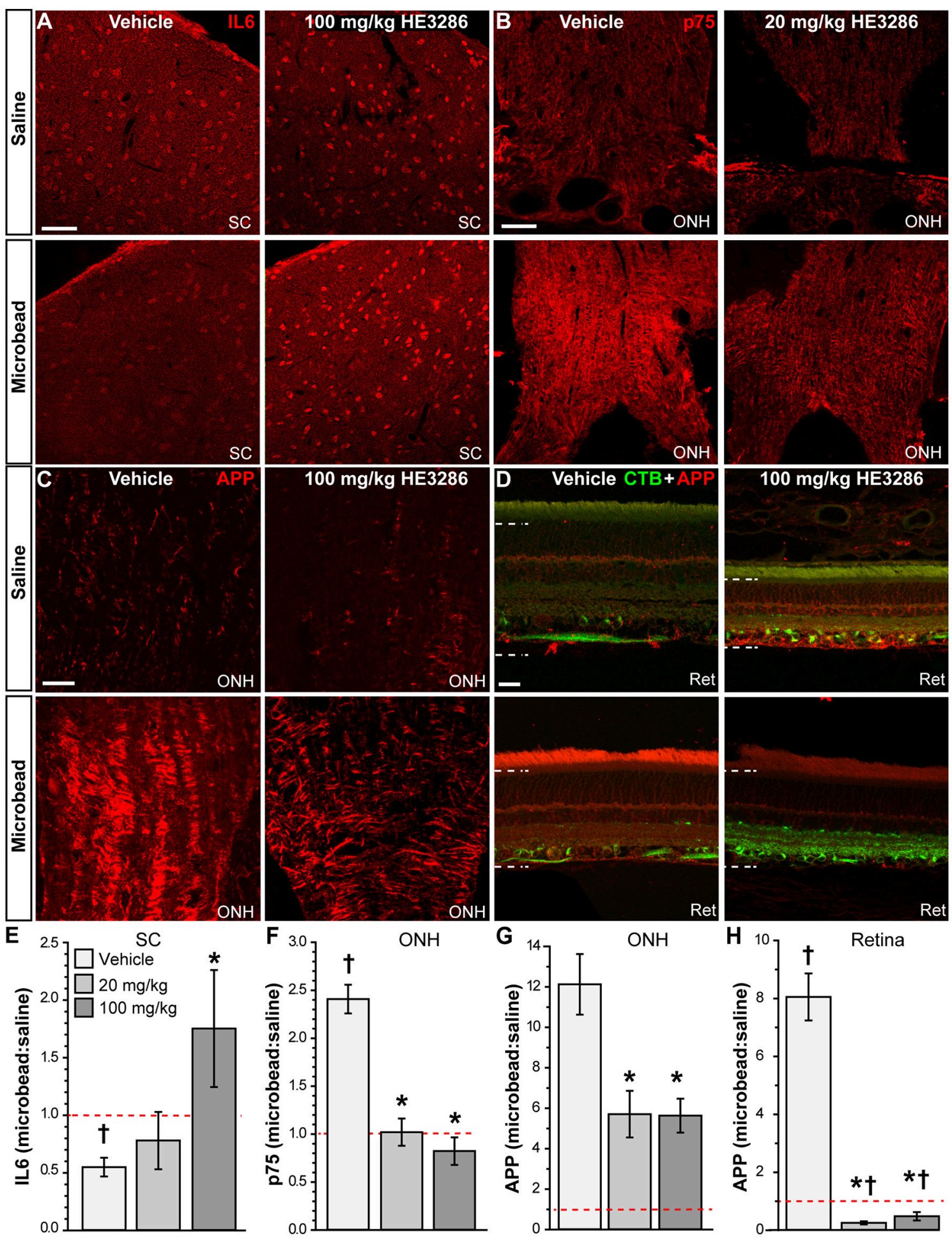

FIGURE 5 | HE3286 treatment influences other degenerative markers in optic projection. Confocal micrographs of saline- and microbead-injected tissue from vehicle and HE3286 rats show immunolabeling for IL6 in superior colliculus (SC, A), p75 in optic nerve head (ONH, B), and APP in optic nerve head (ONH, C) and retina (Ret, D), which also shows RGCs labeled by CTB uptake. Dotted lines indicate region of retina where immunolabel was quantified. Scale: $50 \mu \mathrm{m}$ (A-C) and $20 \mu \mathrm{m}$ (D). Bar graphs (E-H) indicate quantification of each of these markers in the specified tissue expressed as the microbead:saline ratio for vehicle and HE3286 treatment groups. SC: superior colliculus; $\mathrm{ONH}$ : optic nerve head. ${ }^{\dagger}$ Indicates significant departure from expected ratio of unity $(p \leq 0.047)$, which is indicated (dotted line). *indicates $p<0.027$ compared to ratio for vehicle group. $n=6$ animals per vehicle treatment group, 5 animals per 20 mg/kg and 100 mg/kg HE3286 groups for $\mathrm{SC} ; n=3$ animals per treatment group for $\mathrm{ONH}$ and retina analysis. 
TABLE 3 | Significant HE3286-induced changes in expression compared to vehicle.

\begin{tabular}{|c|c|c|c|c|c|}
\hline Target & Tissue & Vehicle ( $p$-value) & $20 \mathrm{mg} / \mathrm{kg}$ & 100 mg/kg & $p$-values \\
\hline $\mathrm{TNF} \alpha$ & $\mathrm{ONH}$ & $1.68 \pm 0.20(0.038)$ & $0.28 \pm 0.10: 83 \% \downarrow$ & No change & 0.003 \\
\hline IL6R $\alpha \mathrm{m}$ & $\mathrm{ONH}$ & $1.93 \pm 0.09(<0.001)$ & $0.72 \pm 0.03: 62 \% \downarrow$ & $0.74 \pm 0.11: 63 \% \downarrow$ & $<0.001$ \\
\hline IL6 & Retina & $5.43 \pm 0.84$ (0.006) & $2.10 \pm 0.37: 61 \% \downarrow$ & $2.55 \pm 0.65: 53 \% \downarrow$ & $\leq 0.045$ \\
\hline p75 & Retina & $2.41 \pm 0.22(0.022)$ & $1.53 \pm 0.20: 37 \% \downarrow$ & $1.26 \pm 0.21: 48 \% \downarrow$ & $\leq 0.041$ \\
\hline CD44 & Retina & $1.32 \pm 0.10$ & No change & $0.48 \pm 0.11: 63 \% \downarrow$ & 0.005 \\
\hline$A \beta$ & Retina & $8.89 \pm 0.58(<0.001)$ & $13.19 \pm 1.33: 48 \% \uparrow$ & $5.02 \pm 0.88: 44 \% \downarrow$ & $\leq 0.042$ \\
\hline
\end{tabular}

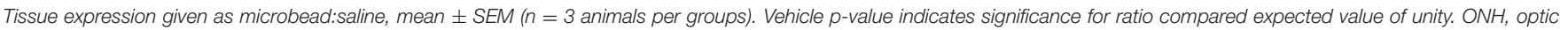

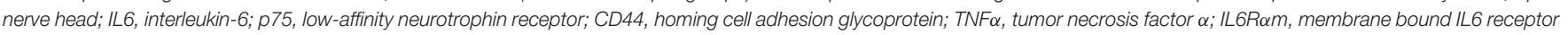
$\alpha$; $A \beta$, beta-amyloid. $\uparrow$, increase compared to vehicle; $\downarrow$, decrease compared to vehicle.

nuclear localization of NFKB in SC, optic nerve head, and retina. In vehicle-treated rats, NFKB localized both within and external to nuclei in SC; microbead SC appeared to have less nuclear localization (Figure 7B). Treatment with HE3286 increased $\mathrm{NF}_{\kappa} \mathrm{B}$ particularly in microbead SC. Within the $\mathrm{ONH}$ (Figure 7C), microbead-induced elevations in IOP increased NFKB in nuclei for the vehicle group; this was prevented with HE3286 treatment. In retinal sections (Figure 7D), elevated IOP had little effect on NFKB localization. However, HE3286 appeared to increase NFKB translocation, particularly in RGCs of microbead retina.

Using an established algorithm (Carmona et al., 2007; Schneider et al., 2012), we quantified the degree of nuclear localization of $\mathrm{NF \kappa B}$ as the ratio of levels in tissue from microbead to saline eyes (Figures 7E-G). For SC (Figure 7E), this ratio was significantly lower than an expected value of 1 for vehicle rats $(p=0.006)$, consistent with our qualitative observation (Figure 7B). Treatment with HE3286 reversed this trend as we found that the ratio of microbead to saline nuclear $\mathrm{NF \kappa B}$ increased $77 \%(1.25 \pm 0.09)$ in rats treated with $20 \mathrm{mg} / \mathrm{kg}$ HE3286 and by $39 \%(0.99 \pm 0.09)$ in rats treated with $100 \mathrm{mg} / \mathrm{kg}$ HE3286; both changes were significant compared to vehicle $(0.71$ $\pm 0.5 ; p \leq 0.031$ ). In the $\mathrm{ONH}$ (Figure 7F), $\mathrm{NF \kappa B}$ in nuclei increased significantly with microbead elevations in IOP for the vehicle group $(1.50 \pm 0.18 ; p=0.047)$. Treatment with 100 $\mathrm{mg} / \mathrm{kg}$ HE3286 decreased the ratio of microbead to saline levels by $35 \%(0.97 \pm 0.12)$, which was significant compared to vehicle $(p=0.038)$. Treatment with $20 \mathrm{mg} / \mathrm{kg}$ HE3286 had little effect compared to vehicle $(1.41 \pm 0.22 ; p=0.753)$. Similar to the $\mathrm{SC}$, nuclear NFKB in the retina increased with IOP elevation for the HE3286 treatment groups (Figure 7G). Treatment with 100 $\mathrm{mg} / \mathrm{kg}$ HE3286 caused a $62 \%$ increase $(1.47 \pm 0.14)$ compared to the microbead:saline ratio in vehicle $(0.90 \pm 0.25 ; p=0.044)$; this was also significantly different from an expected ratio of 1 $(p=0.031)$. The $20 \mathrm{mg} / \mathrm{kg}$ HE3286 dose also increased NFкB nuclear labeling in microbead retina $(1.368 \pm 0.185)$, but the $51 \%$ increase was not significant both compared to vehicle $(p=0.087)$ and to an expected ratio of unity $(p=0.117)$.

$\mathrm{NF \kappa B}$ activation can promote degeneration or survival depending on the cell type (e.g., glia vs. neurons; Mattson and Camandola, 2001). In SC from each treatment group, NFкB localized to nuclei of GFAP-labeled astrocytes (Figure 8A), Iba1labeled microglia (Figure 4B), and neurons labeled by NeuN or pNFH (Figures 8C,D). Whether nuclear localization was glial or neuronal did not appear to be influenced by elevated IOP in the vehicle cohort, since SC from saline and microbead eyes showed both. NFкB localization in neuronal nuclei did appear to increase with elevated IOP in HE3286-treated rats, consistent with the overall increase in $\mathrm{NF \kappa B}$ we observed earlier (Figures 7B,E).

Within the $\mathrm{ONH}$, the majority of $\mathrm{NF \kappa B}$ appeared to localize in the cytoplasm surrounding DAPI-labeled nuclei, though nuclei of some astrocytes (Figure 9A) and microglia (Figure 9B) contained NFKB. Consistent with the overall increase within the ONH (Figures 7C,F), microbead-induced elevated IOP appeared to increase glial NFKB nuclear localization in vehicle rats, which was attenuated by HE3286. In retina, nuclei of RGCs identified by CTB uptake and GFAP-labeled astrocytes also demonstrated NFKB localization (Figures 9C,D). Elevated IOP appeared to decrease nuclear NFKB in vehicle rats, with HE3286 treatment reversing this trend. Again, this finding is consistent with the overall trend of NFKB localization we quantified in retina (Figures 7D,G).

\section{HE3286 Decreased Retinal Thickness in Rats with Induced Ocular Hypertension}

While examining vertical retinal sections following immunohistochemistry, we noted what appeared to be a decrease in retinal thickness within the mid-peripheral region of the retina in rats treated with HE3286 compared to vehicle (Figure 10A). To examine this further, we measured retinal thickness across groups and retinal locations. Comparing mean retinal thickness (Figure 10C) showed that for vehicle-treated and $20 \mathrm{mg} / \mathrm{kg}$ HE3286-treated rats, retinal thickness was decreased in microbead-injected eyes compared to salineinjected eyes, but this difference was not significant (vehicle: $132.51 \pm 1.47$ vs. $130.93 \pm 1.99 \mu \mathrm{m}, p=0.557 ; 20 \mathrm{mg} / \mathrm{kg}$ dose: $116.35 \pm 2.50$ vs. $111.47 \pm 2.11 \mu \mathrm{m}, p=0.21$ ). In contrast, rats treated with $100 \mathrm{mg} / \mathrm{kg} \mathrm{HE} 3286$ had significantly thinner retinas in saline-injected eyes $(87.70 \pm 0.59 \mu \mathrm{m})$ compared to microbead-injected eyes (96.91 $\pm 0.98 \mu \mathrm{m} ; p=0.001)$. Comparing saline-injected eyes across the groups showed that treatment with HE3286 decreased retinal thickness 12-34\% compared to treatment with vehicle $(p<0.005)$. Similarly, retinal thickness decreased $15-26 \%$ in microbead eyes treated with HE3286 compared to vehicle $(p<0.002)$. Retinal thinning was dependent on dosage, as a $13-25 \%$ decrease was observed in eyes that received $100 \mathrm{mg} / \mathrm{kg}$ HE3286 compared to $20 \mathrm{mg} / \mathrm{kg}$ 

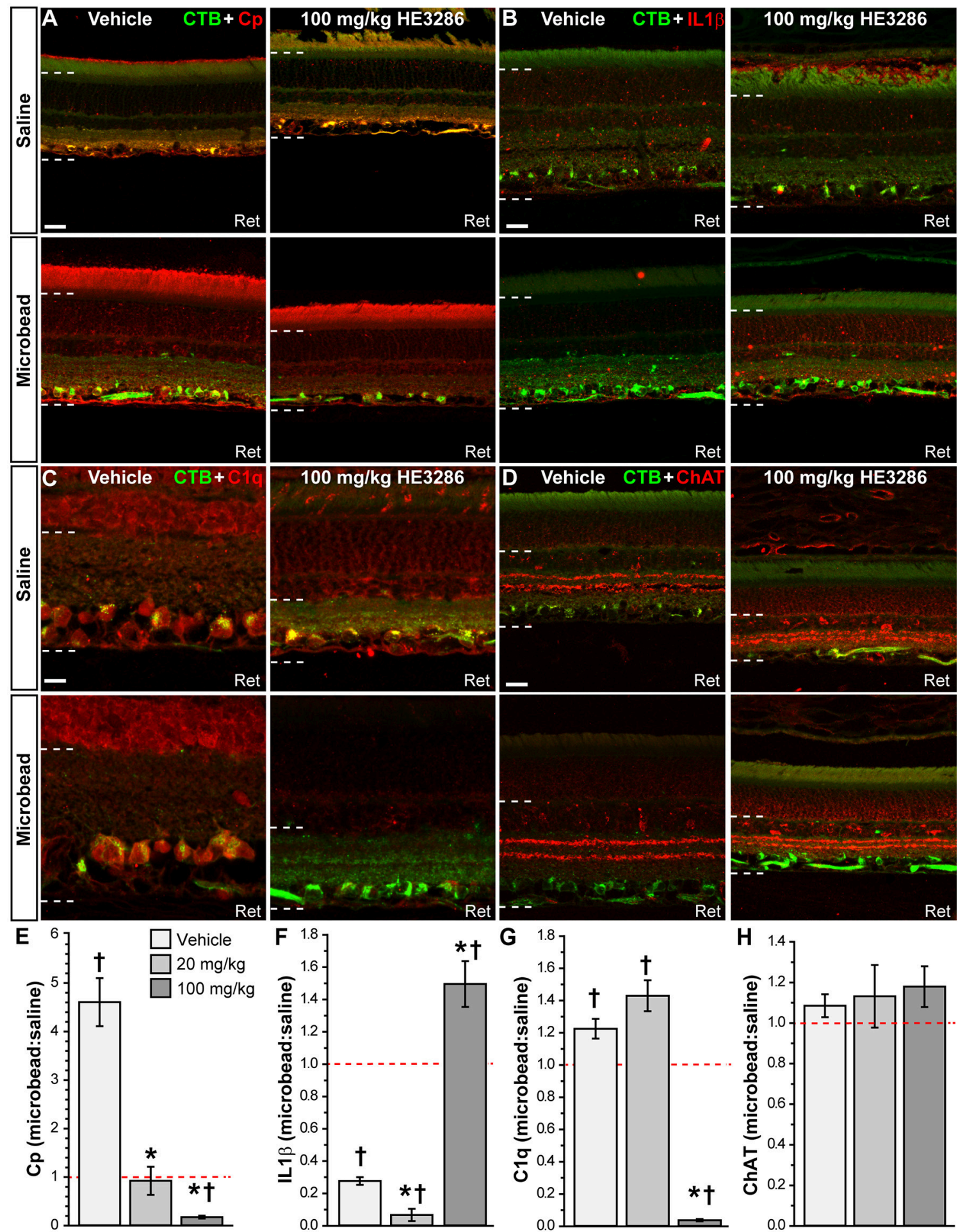

FIGURE 6 | HE3286 treatment influences retinal expression of common pathogenic markers. Confocal micrographs of saline- and microbead-injected from vehicle and HE3286 rats show immunolabeling in retina (Ret) for ceruloplasmin (Cp, A), interleukin $1 \beta$ (IL1 $\beta, \mathbf{B})$, and C1q, the first component of the classical complement pathway (C). Localization of amacrine cell neurons specific for ChAT (choline acetyltransferase) is shown for comparison (D). CTB-labeled RGCs are shown in each panel. Dotted lines indicate region of retina where immunolabel was quantified. Scale: $20 \mu \mathrm{m}$ (A,B,D) or $10 \mu \mathrm{m}$ (C). Bar graphs (E-H) indicate quantification of each of these markers expressed as the microbead:saline ratio for vehicle and HE3286 treatment groups. ${ }^{\dagger}$ Indicates significant departure from expected ratio of unity $(p \leq 0.037)$, which is indicated (dotted line). *indicates $p \leq 0.033$ compared to ratio for vehicle group. $n=3$ animals per treatment group. 

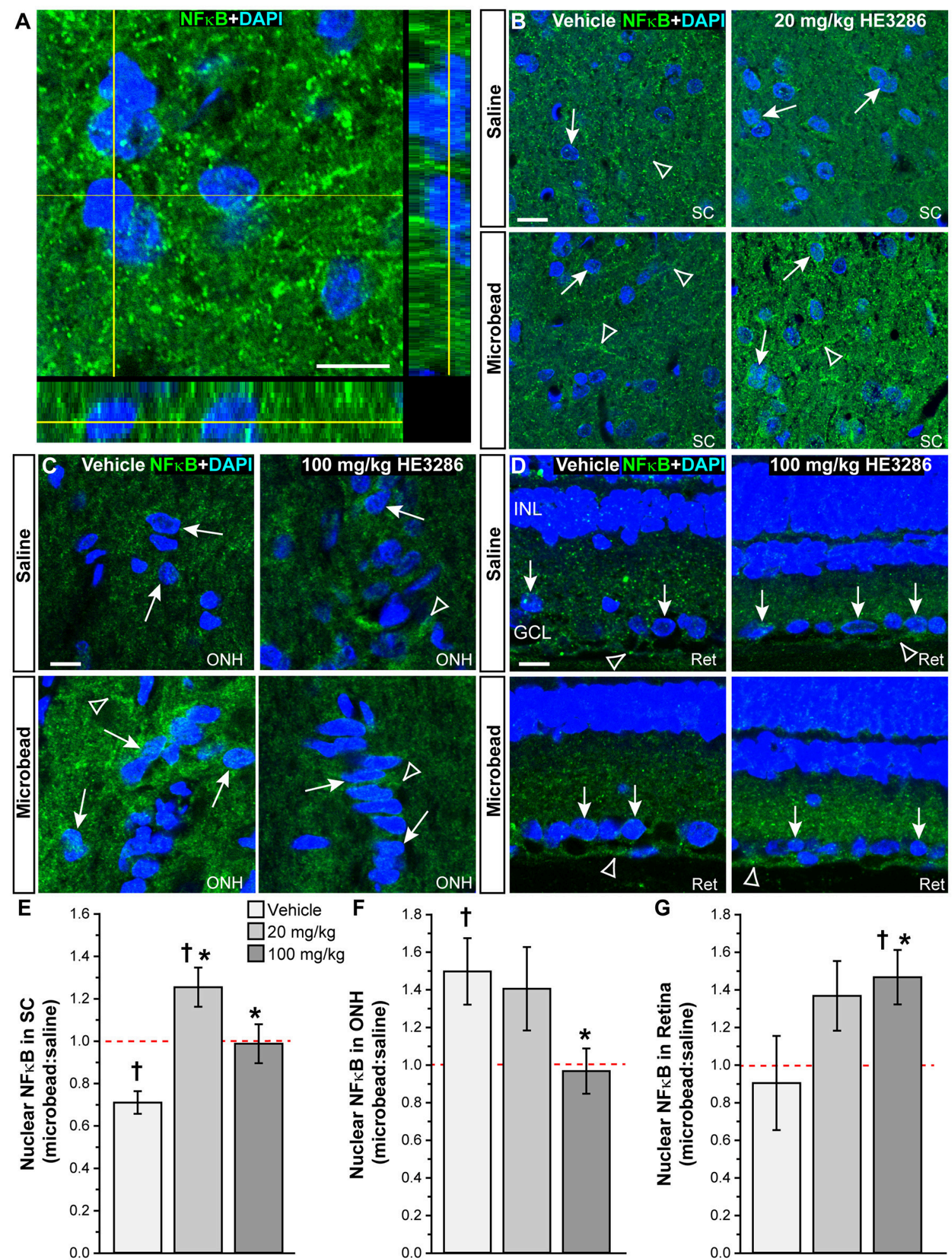

FIGURE 7 | HE3286 modulates NFкB nuclear localization in the optic projection. (A) Confocal micrograph of a coronal section through naïve rat SC shows localization of NFKB (green) both outside and within DAPI-labeled nuclei (blue). Lines indicate location of orthogonal rotations through nuclei. Scale: $10 \mu \mathrm{m}$. Representative images (single optical plane) of superior colliculus (SC, B), optic nerve head (ONH, C) and retina (Ret, D) from vehicle and HE3286 rats shows localization of NFKB (green) both within (arrows) and external to (arrowhead) DAPI-stained nuclei. INL: inner nuclear layer of retina; GCL: ganglion cell layer. Scale: 10 $\mu \mathrm{m}$. Bar graphs show nuclear localization of $\mathrm{NF}_{\kappa} \mathrm{B}$ in $\mathrm{SC}(\mathbf{E})$, optic nerve head $(\mathrm{ONH}, \mathbf{F})$, and retina (G) expressed as the ratio of microbead:saline in vehicle and HE3286 treated tissue; ratio of 1 , or unity, indicated by dotted line. ${ }^{\dagger}$ Indicates significant departure from expected ratio of unity $(p \leq 0.026)$. ${ }^{*}$ Indicates $p \leq 0.044$ compared to ratio for vehicle group. $n=5$ animals per treatment group. 

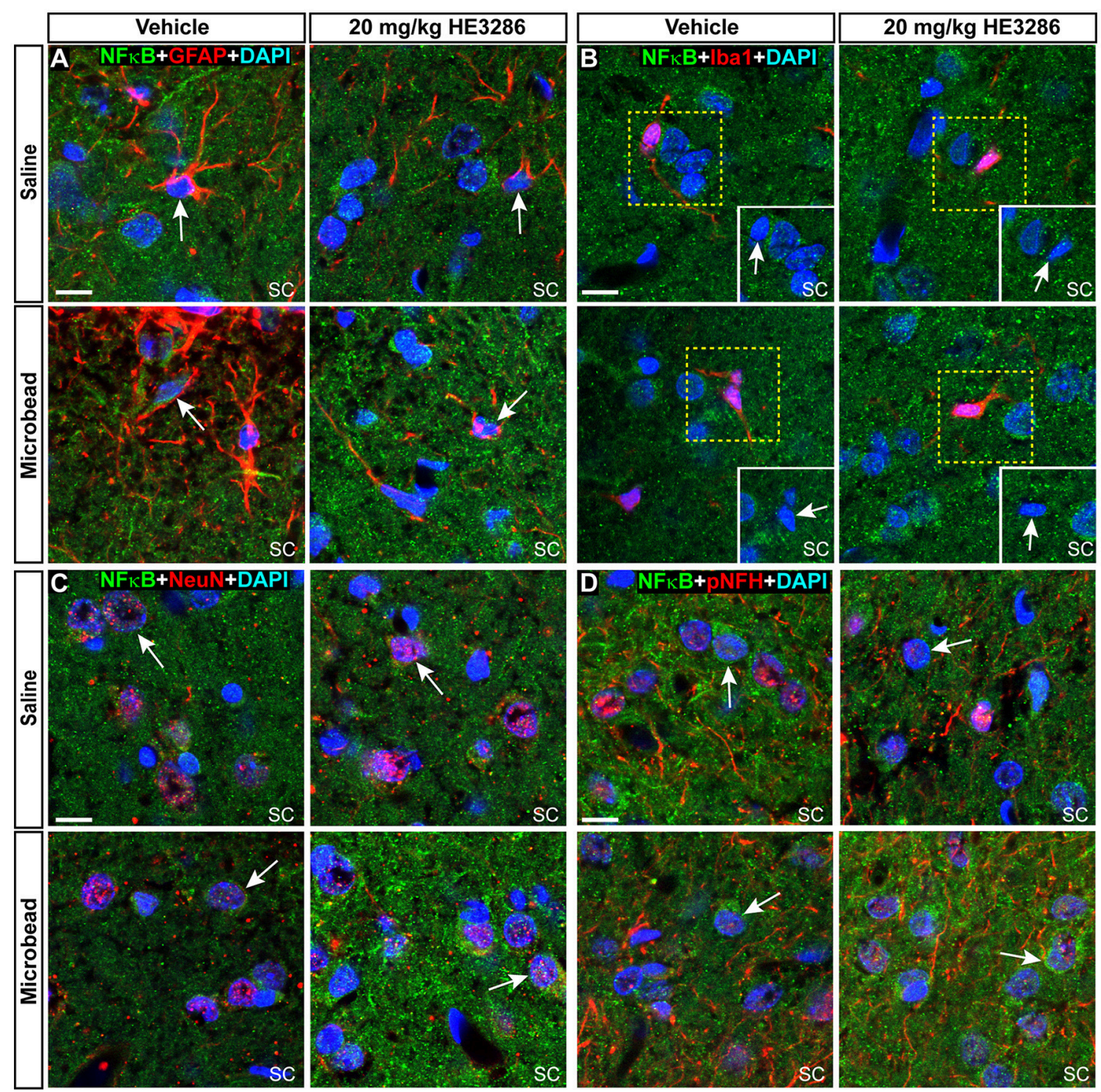

FIGURE 8 | NFKB localizes to both neuronal and glial nuclei in superior colliculus. Representative confocal images of NFKB in the superior colliculus (SC) of vehicle- and HE3286-treated rats with all nuclei indicated (DAPI). Sections were also labeled for astrocytes (A, GFAP), microglia (B, Iba1), or neurons using antibodies against either NeuN (C) or phosphorylated neurofilament heavy (pNFH; D). Representative nuclei from each cell class demonstrated NFkB localization (arrows). Insets (solid white, B) show region contained within dashed box with red channel removed to better visualize nuclear localization of NFKB. Scale: $10 \mu \mathrm{m}$.

$(p<0.003)$. To determine if retinal thinning involved neuronal loss, we counted RGCs that were positive for CTB uptake and DAPI nuclear labeling and measured the thickness of the inner nuclear and outer nuclear layers of the retinas (Figure 10D). Treatment with HE3286 did not reduce the number of RGCs in $20 \mathrm{mg} / \mathrm{kg}\left(3.14 \times 10^{-4} \pm 7.77 \times 10^{-6} \mathrm{RGCs} / \mu \mathrm{m}^{2}\right)$ or $100 \mathrm{mg} / \mathrm{kg}$ $\left(3.16 \times 10^{-4} \pm 7.48 \times 10^{-6} \mathrm{RGCs} / \mu \mathrm{m}^{2}\right)$ HE3296-treated retinas compared to vehicle-treated retinas $\left(3.14 \times 10^{-4} \pm 6.91 \times 10^{-6}\right.$ RGCs $\left./ \mu \mathrm{m}^{2} ; p>0.963\right)$. In contrast, HE3286 treatment reduced INL thickness $16 \%$ for the $20 \mathrm{mg} / \mathrm{kg}$ dose $(14.1 \pm 0.2 \mu \mathrm{m})$ and $21 \%$ for the $100 \mathrm{mg} / \mathrm{kg}$ dose $(13.2 \pm 0.1 \mu \mathrm{m})$ compared to vehicle (16.8 $\pm 0.3 \mathrm{~mm} ; p<0.001)$. This response was dose-dependent, as $100 \mathrm{mg} / \mathrm{kg} \mathrm{HE} 3286$ reduced INL thickness a further 6\% compared to $20 \mathrm{mg} / \mathrm{kg}(p=0.029)$. A similar reduction in ONL thickness was observed at the higher dose of HE3286 (27.1 \pm 0.2 $\mu \mathrm{m})$ compared to vehicle $(33.6 \pm 0.4 \mu \mathrm{m})$ or the $20 \mathrm{mg} / \mathrm{kg}$ dose $(33.4 \pm 0.6 \mu \mathrm{m} ; p>0.001)$

\section{DISCUSSION}

In this study, we examined the therapeutic potential of a sterol derivative, HE3286, in an inducible model of glaucoma. Daily oral gavage with either $20 \mathrm{mg} / \mathrm{kg}$ or $100 \mathrm{mg} / \mathrm{kg} \mathrm{HE} 3286 \mathrm{did}$ not affect IOP following microbead or saline injection. Even 

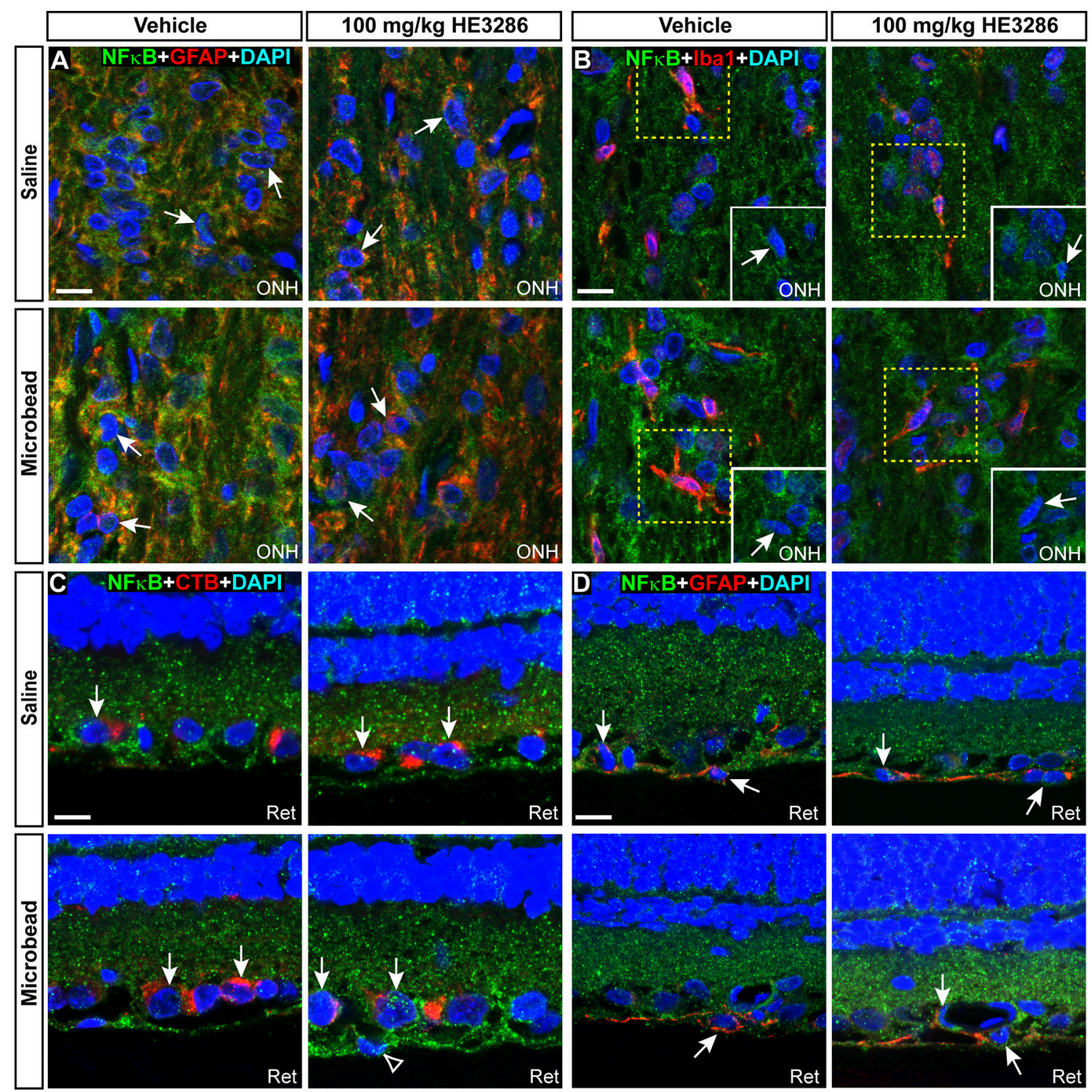

FIGURE 9 | NFKB localizes to glial nuclei in optic nerve head and to glial and neuronal nuclei in retina. Representative confocal images of NFKB (green) localization in DAPI-stained nuclei of astrocytes (A, GFAP) and microglia (B, Iba1) in optic nerve head (ONH) of vehicle- and HE3286-treated rats. Inset in (B) is region contained within dashed box with red channel removed to better visualize nuclear localization of NFKB. In retina (C,D), NFKB localizes to nuclei of RGCs with CTB uptake and GFAP-labeled astrocytes within the retina (Ret). Arrows indicate cells positively-identified by specific label. Scale: $10 \mu \mathrm{m}$.

so, treatment with HE3286 did have tremendous influence on outcomes typically associated with IOP-related axonopathy. We found that HE3286 (1) preserved anterograde axonal transport of CTB from the retina to the superior colliculus in microbeadinjected eyes, (2) countered the influence of elevated IOP throughout the optic projection with respect to levels of BDNF, Ibal and other proteins associated with neuroinflammation and neurodegeneration, (3) modulated pathogenic markers of glaucoma within the retina, (4) increased NFKB localization to neuronal nuclei in the superior colliculus and retina, and (5) decreased NFKB localization to glial nuclei in the optic nerve head.
HE3286 treatment also resulted in weight loss and retinal thinning. In a safety and pharmacokinetic study, Ahlem et al. found that HE3286 had no adverse effects in chronic toxicity studies and was not immunosuppressive (Ahlem et al., 2011). Male rats that received daily oral gavage of 100 to 400 $\mathrm{mg} / \mathrm{kg}$ HE3268 consumed less food and gained less weight than rats treated with vehicle, but showed no corresponding pathology or other effects on general health (Ahlem et al., 2011). We observed a similar weight loss (Table 2), with rats that received HE3286 losing $\sim 6-10 \%$ of their body weight over the course of the study. We observed no adverse effects to general health (e.g., piloerection, hunched posture, decreased 

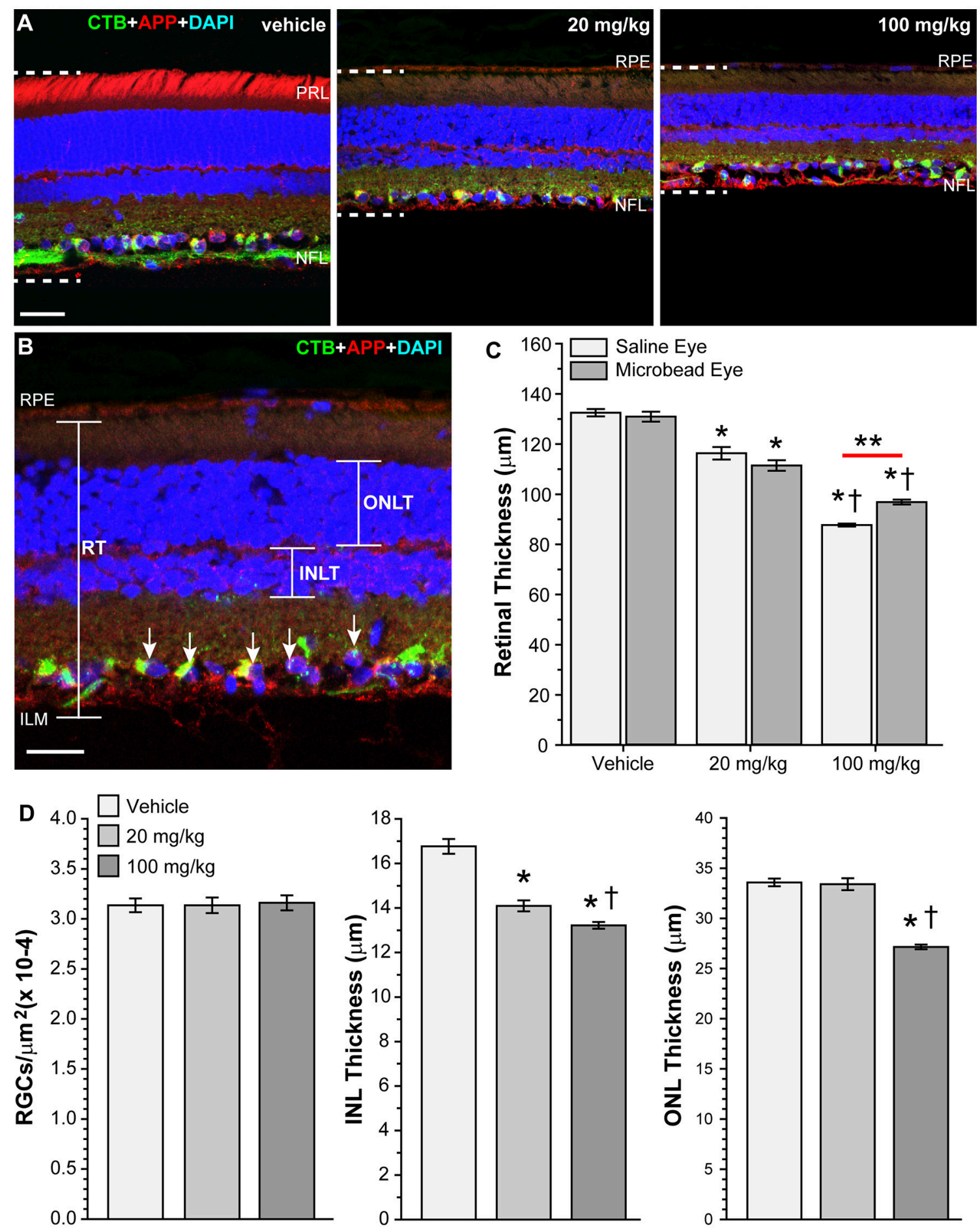

FIGURE 10 | HE3286 treatment decreases retinal thickness. (A) Confocal images of vertical retinal sections from vehicle- and HE3286-treated rats showing CTB uptake (green), immunolabeling for APP (red), and DAPI-labeled nuclei (blue). All images were captured in the mid-peripheral region of the retina. Dotted lines show retinal thickness from nerve fiber layer (NFL) to the retinal pigmented epithelial layer (RPE). Scale bar: $30 \mu \mathrm{m}$. PRL: photoreceptor layer. (B) Higher magnification of retinal section with same markers demonstrating how total retinal thickness (RT), inner nuclear layer thickness (INLT) and outer nuclear layer thickness (ONLT) were measured. RGCs positive for CTB and DAPI (arrows) were counted. Scale bar: $20 \mu \mathrm{m}$. ILM: inner limiting membrane. (C) Bar graph showing HE3286 treatment decreased retinal thickness. ${ }^{*} p<0.005$ compared to vehicle; ${ }^{\dagger} p<0.003$ compared to $20 \mathrm{mg} / \mathrm{kg} \mathrm{HE} 3286 ;{ }^{* *} p=0.0013 ; n=8$ images per animal, three animals per treatment group. (D) Bar graphs showing HE3286 did not reduce RGC number, but did result in thinning of INL and ONL. ${ }^{*} p<0.001$ compared to vehicle; ${ }^{\dagger} p<$ 0.029 compared to $20 \mathrm{mg} / \mathrm{kg} \mathrm{HE} 3286$ for INL thickness. ${ }^{*} p<0.001$ compared to vehicle; ${ }^{\dagger} p<0.001$ compared to $20 \mathrm{mg} / \mathrm{kg} \mathrm{HE3286}$ for ONL thickness. $n=8$ images per animal, three animals per treatment group. 
responsiveness, labored breathing, sudden lethargy, changes in muscle tone) other than weight loss. We did, however, note thinning of the retina and nuclear layers in rats that received HE3286 (Figure 10). Retinal thickness in vehicle treated rats $(\sim 130 \mu \mathrm{m})$ was similar to what has been previously reported (Cavallotti et al., 2001; Jiao et al., 2013). Regardless of microbead treatment, retinas from rats treated with HE3286 were 12$34 \%$ thinner than rats that received vehicle. This could be a side effect of sterol treatment, as Razali et. al. reported reduced retinal thickness in a model of steroid induced ocular hypertension in rats (Razali et al., 2015). However, HE3286 is not a glucocorticoid or glucocorticoid mimetic, nor does it bind the glucocorticoid nuclear hormone receptor (Ahlem et al., 2011), so this explanation is unlikely. Also, HE3286 treatment did not elevate IOP (Figure 1), which other steroids can do (Razeghinejad and Katz, 2012).

Loss of retinal neurons can lead to thinning of the retina and has been observed in many neurodegenerative disorders or their models, including glaucoma, Alzheimer's disease, multiple sclerosis, and Parkinson's disease (Lee et al., 2014; Vidal-Sanz et al., 2015; Gupta et al., 2016; Knier et al., 2016a). The retinal thinning seen following HE3286 treatment is unlikely to be due to RGC loss, as counts of CTB+/DAPI+ cells (RGCs) in the ganglion cell layer showed no significant differences between vehicle- and HE3286-treated retinas. This was not surprising as RGC soma loss occurs late in the progression of glaucoma (Buckingham et al., 2008; Calkins, 2012). We did see significant thinning of the INL and ONL with HE3286 treatment. This could be a sign of effective treatment. Interestingly, thickening of the INL has been noted in multiple sclerosis patients and correlates with inflammatory disease severity and disease progression (Saidha et al., 2015; Knier et al., 2016a). This increase in INL volume is thought to be due to retinal inflammation; reducing inflammation via immunomodulatory therapy results in thinning of the INL (Saidha et al., 2015; Knier et al., 2016a,b). HE3286 treatment decreased levels of inflammatory markers in this study (Figures 4-6, Table 3). Thinning of the INL and ONL by HE3286 (Figure 10) may reflect a lower inflammatory response in these tissues.

In addition to preserving anterograde axonal transport (Figure 2), HE3286 also mitigated other indicators of dysfunction. For example, treatment with HE3286 resulted in a lack of BDNF elevation in the SC (Figures 3A,D). In a previous study, we observed focal increases in BDNF in regions of the SC where anterograde transport was depleted due to elevated IOP (Crish et al., 2013). Upregulation of $\mathrm{BDNF}$ in glaucoma is likely to result from retinorecipient targets responding to glaucoma-relevant stress to improve RGC survival (Chen and Weber, 2004; Lebrun-Julien and Di Polo, 2008; Weber et al., 2010). HE3286's preservation of anterograde transport prevented the need to increase BDNF in the SC, implying that restoration of RGC function (i.e., axonal transport) arrests the complex downstream cascades of gliosis and neuroinflammation normally observed in this condition. Conversely, HE3286 treatment also increased BDNF in the $\mathrm{ONH}$ and retina (Figures 3B-D). Neurotrophic factor deprivation due to impaired neurotrophin transport from the brain to the retina is a longstanding hypothesis in the pathogenesis of RGC degeneration in glaucoma (Johnson et al., 2011). Increased BDNF in the optic nerve and retina with IOP elevations suggests that HE3286 acts on the neurotrophin system, either directly, or indirectly by increasing transport, to preserve RGCs (Crish et al., 2013). Whether HE3296 increases BDNF by affecting the trafficking of BDNF or by stimulating local production of BDNF in the retina and optic nerve remains to be determined (Johnson et al., 2011; Crish and Calkins, 2015).

Treatment with HE3286 modulated other proteins implicated in neuroinflammation or neurodegeneration within the optic projection (Figures 5, 6, Table 3). For certain proteins, one dose of HE3286 appeared more effective than the other; this has been observed previously for HE3286 (Ahlem et al., 2009). HE3286 may be more effective at regulating signaling pathways or maintaining homeostasis in a specific tissue at one dose versa another. Microglia become activated in response to injury and upregulate a variety of proteins, including Iba1 (Kreutzberg, 1996; Ito et al., 1998; Soto and Howell, 2014; Mac Nair and Nickells, 2015). Levels of Ibal were elevated in the SC, ONH, and retina of vehicle-treated rats; this is not surprising since microglial activation has been observed in the optic projection in glaucoma (Wang et al., 2000; Yuan and Neufeld, 2001; Imamura et al., 2009; Ebneter et al., 2010; Shimazawa et al., 2012). HE3286 reduced Iba1 expression in all tissues examined, suggesting it abated microglia activation; a similar effect on microglia was observed in a model of optic neuritis (Khan et al., 2014). Treatment with HE3286 also reduced IL6 levels in the retina and levels of IL6R $\alpha \mathrm{m}$ and TNFo in ONH. Activated microglia produce these pro-inflammatory proteins during glaucoma pathogenesis (Tezel et al., 2001; Sappington and Calkins, 2008; Roh et al., 2012; Sims et al., 2012; Cueva Vargas et al., 2015; Wilson et al., 2015), so it's not unexpected that HE3286 would curb their expression in our model. Interestingly, HE3286 increased levels of IL6 within the SC and IL1 $\beta$ in the retina. While associated with neuroinflammation and implicated in neurodegeneration, both IL6 and IL1 $\beta$ have been shown to be neuroprotective under certain conditions (Sappington et al., 2006; Biber et al., 2008; Chidlow et al., 2012; Matousek et al., 2012; Noguchi et al., 2013; Song et al., 2013; Madeira et al., 2015; Wilson et al., 2015). HE3286 also increased APP levels in saline-injected eyes (Figure 5D). HE3286 is a synthetic derivative of a DHEA metabolite (Ahlem et al., 2011). DHEA levels have been linked to Alzheimer's disease, but the exact role of this neurosteroid in disease progression is currently under debate (Kim et al., 2003; Naylor et al., 2008; Aldred and Mecocci, 2010; Hampl and Bicikova, 2010; Rasmuson et al., 2011). DHEA treatment was neuroprotective in a rat model of $\mathrm{AD}$, and in vitro DHEA increases the expression of APP and its cleavage to non-amyloidogenic fragments (Danenberg et al., 1996; Aly et al., 2011). It is possible the increase in APP levels observed in HE3286-treated saline-injected eyes is the result of DHEA-like APP processing. APP can be neuroprotective in some cases (Yankner et al., 1989; Schubert and Behl, 1993; Mucke et al., 1994; Allinquant et al., 1995; Zheng and Koo, 2011). Future studies could include elucidating the effects of HE3286 on APP and its processing in glaucoma and other 
neurodegenerative diseases. Finally, HE3286 decreased APP and p75 in the $\mathrm{ONH}$ and retina, and dramatically reduced ceruloplasmin and $\mathrm{C1q}$ expression in the retina of microbeadinjected eyes. Each of these factors has been implicated in RGC death, or has been shown to be elevated in the retina and optic nerve in human glaucomatous tissue and/or in models of glaucoma (Stasi et al., 2006, 2007; Goldblum et al., 2007; Stevens et al., 2007; Wei et al., 2007; Lebrun-Julien et al., 2009; Kipfer-Kauer et al., 2010; Howell et al., 2011, 2014; Ding et al., 2012; Meeker and Williams, 2015). Taken as a whole, it appears HE3286 mitigates the neuroinflammatory response by dampening microglial activation.

The expression of TNF $\alpha$, IL6, APP in neurons and glia, as well as the production of $\beta$-amyloid, and the activation of microglia can all be regulated by $\mathrm{NF} \kappa \mathrm{B}$ activation (Mattson and Camandola, 2001; Camandola and Mattson, 2007). A suggested mechanism of action for HE3286 is the regulation of NFKB activity (Auci et al., 2007; Ahlem et al., 2009, 2011; Offner et al., 2009). NFאB is a transcription factor activated by a wide range of stimuli that in turn regulates the expression of genes involved inflammation, immune response, cell survival and cell death (Gilmore, 2008; Hayden and Ghosh, 2008; Lawrence, 2009). Activated NFKB translocates to the nucleus, and we observed nuclear localization of $\mathrm{NF} \kappa \mathrm{B}$ in neurons and glia within the optic projection of vehicle- and HE3286treated rats following IOP elevation (Figures 7-9). Similar activation of $\mathrm{NF \kappa B}$ in the visual pathway has been observed following injury and in models of glaucoma (Choi et al., 1998; Agapova et al., 2006; Haenold et al., 2014). Treatment with HE3286 increased NFKB localization in neuronal nuclei within the SC and retina and decreased NFкB localization in $\mathrm{ONH}$ glial nuclei in microbead-injected eyes. This cellspecific NFKB activation may explain the beneficial outcomes observed in HE3286-treated rats; NFKB activation in glial cells promotes neuronal degeneration, while activation in neurons promotes survival (Mattson and Camandola, 2001; Camandola and Mattson, 2007). Activated NFкB can enhance mitochondrial bioenergetics and prevent peripheral neuropathy in rodent models of diabetes (Saleh et al., 2013). Increased NFKB localization to RGC nuclei in HE3286-treated microbeadinjected eyes may protect these neurons metabolically (Kong et al., 2009; Baltan et al., 2010; Lee et al., 2011; Coughlin et al., 2015; Kleesattel et al., 2015; Fahy et al., 2016), such that axonal transport is preserved compared to vehicle-treated eyes (Figure 2). Whether or not HE3286 maintains RGC functionality could be assessed using outcomes such as visual evoked potentials, electroretinogram, or two-alternative forcedchoice visual behavioral testing in this or other models of glaucoma.

HE3286 has produced therapeutic benefits in various models of inflammatory disease, in a model of Parkinson's and in clinical trials for diabetic complications (Auci et al., 2007, 2010; Ahlem et al., 2009; Offner et al., 2009; Conrad et al., 2010; Lu et al., 2010; Kosiewicz et al., 2011; Nicoletti et al., 2012; Reading et al., 2013a,b; Khan et al., 2014). HE3286 has been well tolerated and has presented no adverse effects in human trials (Reading et al., 2013a,b). We observed positive therapeutic outcomes in terms of anterograde axonal transport, the expression of proteins associated with neuroinflammation, neurodegeneration, and glaucoma pathogenesis, and $\mathrm{NF \kappa B}$ activation. The results of this study suggest HE3286 provides neuroprotection to RGCs and their axons, which helps preserve function in these neurons following exposure to elevated ocular pressure. This neuroprotection could be due to caloric restriction (Table 2), as has been observed in a mouse model of glaucoma (Guo et al., 2016). However, HE3286's anti-inflammatory activity has been observed in vitro and in mice without weight loss compared to vehicle controls, and in humans without caloric restriction or weight loss (Wang et al., 2010; Reading et al., 2013b). A matched feeding experimental design would be needed to understand the potential contribution of reduced caloric intake in our glaucoma model, but prior evidence in other models suggest the current results are not predominately a result of caloric restriction. Glaucoma shares many common features with other neurodegenerative diseases (Crish and Calkins, 2011; Mckinnon, 2012; Ghiso et al., 2013; Jindal, 2013; Danesh-Meyer and Levin, 2015; Jain and Aref, 2015). Given the number of people currently with or likely to develop glaucoma in the near future, the commonalities between glaucoma and other agerelated neurodegenerative diseases, and that HE3286 is already being tested in clinical trials, further studies on HE3286 and the mechanism of its action in the central nervous system is warranted.

\section{AUTHOR CONTRIBUTIONS}

WL contributed substantially to the experimental design, the acquisition, analysis and interpretation of data, and the writing and revising the manuscript. BC, CF contributed substantially to the acquisition and analysis of data and assisted in manuscript revisions. RS contributed substantially to the experimental design with regards to neuroinflammation, and assisted in the critical revision of the manuscript. CA contributed substantially to the conception and experimental design, and assisted in the critical revision of the manuscript. DC contributed substantially to the conception and experimental design, interpretation of data, and assisted in the critical revision of the manuscript.

\section{FUNDING}

The authors declare that this research was supported by a grant from Harbor Therapeutics, Inc. (San Diego, CA). We also acknowledge support from the Vanderbilt Vision Research Center (5P30EY008126, DC).

\section{ACKNOWLEDGMENTS}

Confocal imaging was performed through the use of the VUMC Cell Imaging Shared Resource (supported by NIH grants CA68485, DK20593, DK58404, DK59637, and EY08126). 


\section{REFERENCES}

Agapova, O. A., Kaufman, P. L., and Hernandez, M. R. (2006). Androgen receptor and NFkB expression in human normal and glaucomatous optic nerve head astrocytes in vitro and in experimental glaucoma. Exp. Eye Res. 82, 1053-1059. doi: 10.1016/j.exer.2005.10.021

Ahlem, C., Auci, D., Mangano, K., Reading, C., Frincke, J., Stickney, D., et al. (2009). HE3286: a novel synthetic steroid as an oral treatment for autoimmune disease. Ann. N. Y. Acad. Sci. 1173, 781-790. doi: 10.1111/j.1749-6632.2009.04798.x

Ahlem, C. N., Kennedy, M. R., Page, T. M., Reading, C. L., White, S. K., Mckenzie, J. J., et al. (2011). Studies of the pharmacology of $17 \alpha$-ethynyl-androst-5-ene$3 \beta, 7 \beta, 17 \beta$-triol, a synthetic anti-inflammatory androstene. Int. J. Clin. Exp. Med. 4, 119-135.

Aldred, S., and Mecocci, P. (2010). Decreased dehydroepiandrosterone (DHEA) and dehydroepiandrosterone sulfate (DHEAS) concentrations in plasma of Alzheimer's disease (AD) patients. Arch. Gerontol. Geriatr. 51, e16-e18. doi: 10.1016/j.archger.2009.07.001

Allinquant, B., Hantraye, P., Mailleux, P., Moya, K., Bouillot, C., and Prochiantz, A. (1995). Downregulation of amyloid precursor protein inhibits neurite outgrowth in vitro. J. Cell Biol. 128, 919-927. doi: 10.1083/jcb.128.5.919

Aly, H. F., Metwally, F. M., and Ahmed, H. H. (2011). Neuroprotective effects of dehydroepiandrosterone (DHEA) in rat model of Alzheimer's disease. Acta Biochim. Pol. 58, 513-520.

Auci, D., Kaler, L., Subramanian, S., Huang, Y., Frincke, J., Reading, C., et al. (2007). A new orally bioavailable synthetic androstene inhibits collageninduced arthritis in the mouse: androstene hormones as regulators of regulatory T cells. Ann. N. Y. Acad. Sci. 1110, 630-640. doi: 10.1196/annals.1423.066

Auci, D. L., Mangano, K., Destiche, D., White, S. K., Huang, Y., Boyle, D., et al. (2010). Oral treatment with HE3286 ameliorates disease in rodent models of rheumatoid arthritis. Int. J. Mol. Med. 25, 625-633. doi: 10.3892/ijmm_00000385

Baltan, S., Inman, D. M., Danilov, C. A., Morrison, R. S., Calkins, D. J., and Horner, P. J. (2010). Metabolic vulnerability disposes retinal ganglion cell axons to dysfunction in a model of glaucomatous degeneration. J. Neurosci. 30, 5644-5652. doi: 10.1523/JNEUROSCI.5956-09.2010

Biber, K., Pinto-Duarte, A., Wittendorp, M. C., Dolga, A. M., Fernandes, C. C., Von Frijtag Drabbe Kunzel, J., et al. (2008). Interleukin-6 upregulates neuronal adenosine Al receptors: implications for neuromodulation and neuroprotection. Neuropsychopharmacology 33, 2237-2250. doi: $10.1038 /$ sj.npp.1301612

Buckingham, B. P., Inman, D. M., Lambert, W., Oglesby, E., Calkins, D. J., Steele, M. R., et al. (2008). Progressive ganglion cell degeneration precedes neuronal loss in a mouse model of glaucoma. J. Neurosci. 28, 2735-2744. doi: 10.1523/JNEUROSCI.4443-07.2008

Calkins, D. J. (2012). Critical pathogenic events underlying progression of neurodegeneration in glaucoma. Prog. Retin. Eye Res. 31, 702-719. doi: 10.1016/j.preteyeres.2012.07.001

Camandola, S., and Mattson, M. P. (2007). NF- $\kappa$ B as a therapeutic target in neurodegenerative diseases. Expert Opin. Ther. Targets 11, 123-132. doi: $10.1517 / 14728222.11 .2 .123$

Carmona, R., Macias, D., Guadix, J. A., Portillo, V., Perez-Pomares, J. M., and Munoz-Chapuli, R. (2007). A simple technique of image analysis for specific nuclear immunolocalization of proteins. J. Microsc. 225, 96-99. doi: 10.1111/j.1365-2818.2007.01719.x

Cavallotti, C., Artico, M., Pescosolido, N., and Feher, J. (2001). Age-related changes in rat retina. Jpn. J. Ophthalmol. 45, 68-75. doi: 10.1016/S0021-5155(00)00294-X

Chen, H., and Weber, A. J. (2004). Brain-derived neurotrophic factor reduces TrkB protein and mRNA in the normal retina and following optic nerve crush in adult rats. Brain Res. 1011, 99-106. doi: 10.1016/j.brainres.2004.03.024

Cheung, W., Guo, L., and Cordeiro, M. F. (2008). Neuroprotection in glaucoma: drug-based approaches. Optom. Vis. Sci. 85, 406-416. doi: 10.1097/OPX.0b013e31817841e5

Chidlow, G., Wood, J. P., Ebneter, A., and Casson, R. J. (2012). Interleukin-6 is an efficacious marker of axonal transport disruption during experimental glaucoma and stimulates neuritogenesis in cultured retinal ganglion cells. Neurobiol. Dis. 48, 568-581. doi: 10.1016/j.nbd.2012.07.026
Choi, J. S., Sungjoo, K. Y., and Joo, C. K. (1998). NF-kB activation following optic nerve transection. Korean J. Ophthalmol. 12, 19-24. doi: 10.3341/kjo.1998.12.1.19

Conrad, D., Wang, A., Pieters, R., Nicoletti, F., Mangano, K., Van Heeckeren, A. M., et al. (2010). HE3286, an oral synthetic steroid, treats lung inflammation in mice without immune suppression. J. Inflamm. 7:52. doi: 10.1186/1476-9255-7-52

Coughlin, L., Morrison, R. S., Horner, P. J., and Inman, D. M. (2015). Mitochondrial morphology differences and mitophagy deficit in murine glaucomatous optic nerve. Invest. Ophthalmol. Vis. Sci. 56, 1437-1446. doi: $10.1167 /$ iovs.14-16126

Crish, S. D., and Calkins, D. J. (2011). Neurodegeneration in glaucoma: progression and calcium-dependent intracellular mechanisms. Neuroscience 176, 1-11. doi: 10.1016/j.neuroscience.2010.12.036

Crish, S. D., and Calkins, D. J. (2015). Central visual pathways in glaucoma: evidence for distal mechanisms of neuronal self-repair. J. Neuroophthalmol. 35(Suppl. 1), S29-S37. doi: 10.1097/wno.0000000000000291

Crish, S. D., Dapper, J. D., Macnamee, S. E., Balaram, P., Sidorova, T. N., Lambert, W. S., et al. (2013). Failure of axonal transport induces a spatially coincident increase in astrocyte BDNF prior to synapse loss in a central target. Neuroscience 229, 55-70. doi: 10.1016/j.neuroscience.2012.10.069

Crish, S. D., Sappington, R. M., Inman, D. M., Horner, P. J., and Calkins, D. J. (2010). Distal axonopathy with structural persistence in glaucomatous neurodegeneration. Proc. Natl. Acad. Sci. U.S.A. 107, 5196-5201. doi: 10.1073/pnas.0913141107

Cueva Vargas, J. L., Osswald, I. K., Unsain, N., Aurousseau, M. R., Barker, P. A., Bowie, D., et al. (2015). Soluble tumor necrosis factor alpha promotes retinal ganglion cell death in glaucoma via calciumpermeable ampa receptor activation. J. Neurosci. 35, 12088-12102. doi: 10.1523/JNEUROSCI.1273-15.2015

Danenberg, H. D., Haring, R., Fisher, A., Pittel, Z., Gurwitz, D., and Heldman, E. (1996). Dehydroepiandrosterone (DHEA) increases production and release of Alzheimer's amyloid precursor protein. Life Sci. 59, 1651-1657. doi: 10.1016/0024-3205(96)00496-1

Danesh-Meyer, H. V., and Levin, L. A. (2015). Glaucoma as a neurodegenerative disease. J Neuroophthalmol 35(Suppl. 1), S22-S28. doi: $10.1097 /$ wno. 0000000000000293

Dapper, J. D., Crish, S. D., Pang, I. H., and Calkins, D. J. (2013). Proximal inhibition of p38 MAPK stress signaling prevents distal axonopathy. Neurobiol. Dis. 59, 26-37. doi: 10.1016/j.nbd.2013.07.001

Dengler-Crish, C. M., Smith, M. A., Inman, D. M., Wilson, G. N., Young, J. W., and Crish, S. D. (2014). Anterograde transport blockade precedes deficits in retrograde transport in the visual projection of the DBA/2J mouse model of glaucoma. Front. Neurosci. 8:290. doi: 10.3389/fnins.2014.00290

Denko, C. W. (1979). Protective role of ceruloplasmin in inflammation. Agents Actions 9, 333-336. doi: 10.1007/BF01970657

Dillon, J. S. (2005). Dehydroepiandrosterone, dehydroepiandrosterone sulfate and related steroids: their role in inflammatory, allergic and immunological disorders. Curr. Drug Targets Inflamm. Allergy 4, 377-385. doi: $10.2174 / 1568010054022079$

Ding, Q. J., Cook, A. C., Dumitrescu, A. V., and Kuehn, M. H. (2012). Lack of immunoglobulins does not prevent $\mathrm{C} 1 \mathrm{q}$ binding to RGC and does not alter the progression of experimental glaucoma. Invest. Ophthalmol. Vis. Sci. 53, 6370-6377. doi: 10.1167/iovs.12-10442

Ebneter, A., Casson, R. J., Wood, J. P., and Chidlow, G. (2010). Microglial activation in the visual pathway in experimental glaucoma: spatiotemporal characterization and correlation with axonal injury. Invest. Ophthalmol. Vis. Sci. 51, 6448-6460. doi: 10.1167/iovs.10-5284

Fahy, E. T., Chrysostomou, V., and Crowston, J. G. (2016). Mini-Review: impaired axonal transport and glaucoma. Curr. Eye Res. 41, 273-283. doi: 10.3109/02713683.2015.1037924

Feng, L., Xie, X., Joshi, P. S., Yang, Z., Shibasaki, K., Chow, R. L., et al. (2006). Requirement for Bhlhb5 in the specification of amacrine and cone bipolar subtypes in mouse retina. Development 133, 4815-4825. doi: 10.1242/dev.02664

Ghiso, J. A., Doudevski, I., Ritch, R., and Rostagno, A. A. (2013). Alzheimer's disease and glaucoma: mechanistic similarities and differences. J. Glaucoma 22(Suppl. 5), S36-S38. doi: 10.1097/ijg.0b013e3182934af6

Gilmore, T. D. (2008). NF-kB Transcription Factors. Available online at: www.NF-kB.org (Accessed). 
Goldblum, D., Kipfer-Kauer, A., Sarra, G. M., Wolf, S., and Frueh, B. E. (2007). Distribution of amyloid precursor protein and amyloid-beta immunoreactivity in DBA/2J glaucomatous mouse retinas. Invest. Ophthalmol. Vis. Sci. 48, 5085-5090. doi: 10.1167/iovs.06-1249

Goldstein, I. M., Kaplan, H. B., Edelson, H. S., and Weissmann, G. (1979). A new function for ceruloplasmin as an acute-phase reactant in inflammation: a scavenger of superoxide anion radicals. Trans. Assoc. Am. Physicians 92, 360-369.

Guo, X., Kimura, A., Azuchi, Y., Akiyama, G., Noro, T., Harada, C., et al. (2016). Caloric restriction promotes cell survival in a mouse model of normal tension glaucoma. Sci. Rep. 6:33950. doi: 10.1038/srep33950

Gupta, V. K., Chitranshi, N., Gupta, V. B., Golzan, M., Dheer, Y., Wall, R. $\mathrm{V}$., et al. (2016). Amyloid beta accumulation and inner retinal degenerative changes in Alzheimer's disease transgenic mouse. Neurosci. Lett. 623, 52-56. doi: 10.1016/j.neulet.2016.04.059

Haenold, R., Weih, F., Herrmann, K. H., Schmidt, K. F., Krempler, K., Engelmann, C., et al. (2014). NF- $\kappa B$ controls axonal regeneration and degeneration through cell-specific balance of RelA and p50 in the adult CNS. J. Cell Sci. 127, 3052-3065. doi: 10.1242/jcs.140731

Hampl, R., and Bicikova, M. (2010). Neuroimmunomodulatory steroids in Alzheimer dementia. J. Steroid Biochem. Mol. Biol. 119, 97-104. doi: 10.1016/j.jsbmb.2010.02.007

Hayden, M. S., and Ghosh, S. (2008). Shared principles in NF-кB signaling. Cell 132, 344-362. doi: 10.1016/j.cell.2008.01.020

Heijl, A., Leske, M. C., Bengtsson, B., Hyman, L., and Hussein, M. (2002). Reduction of intraocular pressure and glaucoma progression: results from the early manifest glaucoma trial. Arch. Ophthalmol. 120, 1268-1279. doi: 10.1001/archopht.120.10.1268

Herkenham, M., Rathore, P., Brown, P., and Listwak, S. J. (2011). Cautionary notes on the use of NF- $\mathrm{B}$ p 65 and p50 antibodies for CNS studies. J. Neuroinflammation 8:141. doi: 10.1186/1742-2094-8-141

Hernandez, M. R. (2000). The optic nerve head in glaucoma: role of astrocytes in tissue remodeling. Prog. Retin. Eye Res. 19, 297-321. doi: 10.1016/S1350-9462(99)00017-8

Hofbauer, A., and Drager, U. C. (1985). Depth segregation of retinal ganglion cells projecting to mouse superior colliculus. J. Comp. Neurol. 234, 465-474. doi: 10.1002/cne.902340405

Howell, G. R., Macalinao, D. G., Sousa, G. L., Walden, M., Soto, I., Kneeland, S. C., et al. (2011). Molecular clustering identifies complement and endothelin induction as early events in a mouse model of glaucoma. J. Clin. Invest. 121, 1429-1444. doi: 10.1172/JCI44646

Howell, G. R., Macnicoll, K. H., Braine, C. E., Soto, I., Macalinao, D. G., Sousa, G. L., et al. (2014). Combinatorial targeting of early pathways profoundly inhibits neurodegeneration in a mouse model of glaucoma. Neurobiol. Dis. 71, 44-52. doi: 10.1016/j.nbd.2014.07.016

Imamura, K., Onoe, H., Shimazawa, M., Nozaki, S., Wada, Y., Kato, K., et al. (2009). Molecular imaging reveals unique degenerative changes in experimental glaucoma. Neuroreport 20, 139-144. doi: 10.1097/WNR.0b013e32831d7f82

Ito, D., Imai, Y., Ohsawa, K., Nakajima, K., Fukuuchi, Y., and Kohsaka, S. (1998). Microglia-specific localisation of a novel calcium binding protein, Iba1. Brain Res. Mol. Brain Res. 57, 1-9. doi: 10.1016/S0169-328X(98)00040-0

Jain, S., and Aref, A. A. (2015). Senile dementia and glaucoma: evidence for a common link. J. Ophthalmic Vis. Res. 10, 178-183. doi: 10.4103/2008-322X.163766

Jiao, J., Mo, B., Wei, H., and Jiang, Y. R. (2013). Comparative study of laserinduced choroidal neovascularization in rats by paraffin sections, frozen sections and high-resolution optical coherence tomography. Graefes Arch. Clin. Exp. Ophthalmol. 251, 301-307. doi: 10.1007/s00417-012-2204-4

Jindal, V. (2013). Glaucoma: an extension of various chronic neurodegenerative disorders. Mol. Neurobiol. 48, 186-189. doi: 10.1007/s12035-013-8416-8

Johnson, T. V., Bull, N. D., and Martin, K. R. (2011). Neurotrophic factor delivery as a protective treatment for glaucoma. Exp. Eye Res. 93, 196-203. doi: 10.1016/j.exer.2010.05.016

Kang, T. H., Ryu, Y. H., Kim, I. B., Oh, G. T., and Chun, M. H. (2004). Comparative study of cholinergic cells in retinas of various mouse strains. Cell Tissue Res. 317, 109-115. doi: 10.1007/s00441-004-0907-5

Khan, R. S., Dine, K., Luna, E., Ahlem, C., and Shindler, K. S. (2014). HE3286 reduces axonal loss and preserves retinal ganglion cell function in experimental optic neuritis. Invest. Ophthalmol. Vis. Sci. 55, 5744-5751. doi: $10.1167 /$ iovs.14-14672

Kim, S. B., Hill, M., Kwak, Y. T., Hampl, R., Jo, D. H., and Morfin, R. (2003). Neurosteroids: cerebrospinal fluid levels for Alzheimer's disease and vascular dementia diagnostics. J. Clin. Endocrinol. Metab. 88, 5199-5206. doi: 10.1210/jc.2003-030646

Kipfer-Kauer, A., Mckinnon, S. J., Frueh, B. E., and Goldblum, D. (2010). Distribution of amyloid precursor protein and amyloid-beta in ocular hypertensive C57BL/6 mouse eyes. Curr. Eye Res. 35, 828-834. doi: $10.3109 / 02713683.2010 .494240$

Kleesattel, D., Crish, S. D., and Inman, D. M. (2015). Decreased energy capacity and increased autophagic activity in optic nerve axons with defective anterograde transport. Invest. Ophthalmol. Vis. Sci. 56, 8215-8227. doi: $10.1167 /$ iovs.15-17885

Knier, B., Berthele, A., Buck, D., Schmidt, P., Zimmer, C., Muhlau, M., et al. (2016a). Optical coherence tomography indicates disease activity prior to clinical onset of central nervous system demyelination. Mult. Scler. 22, 893-900. doi: $10.1177 / 1352458515604496$

Knier, B., Schmidt, P., Aly, L., Buck, D., Berthele, A., Muhlau, M., et al. (2016b). Retinal inner nuclear layer volume reflects response to immunotherapy in multiple sclerosis. Brain 139, 2855-2863. doi: 10.1093/brain/aww219

Kong, G. Y., Van Bergen, N. J., Trounce, I. A., and Crowston, J. G. (2009). Mitochondrial dysfunction and glaucoma. J. Glaucoma 18, 93-100. doi: 10.1097/IJG.0b013e318181284f

Kosiewicz, M. M., Auci, D. L., Fagone, P., Mangano, K., Caponnetto, S., Tucker, C. F., et al. (2011). HE3286, an orally bioavailable synthetic analogue of an active DHEA metabolite suppresses spontaneous autoimmune diabetes in the non-obese diabetic (NOD) mouse. Eur. J. Pharmacol. 658, 257-262. doi: 10.1016/j.ejphar.2011.02.016

Kreutzberg, G. W. (1996). Microglia: a sensor for pathological events in the CNS. Trends Neurosci. 19, 312-318. doi: 10.1016/0166-2236(96)10049-7

Lambert, W. S., Ruiz, L., Crish, S. D., Wheeler, L. A., and Calkins, D. J. (2011). Brimonidine prevents axonal and somatic degeneration of retinal ganglion cell neurons. Mol. Neurodegener. 6:4. doi: 10.1186/1750-1326-6-4

Lawrence, T. (2009). The nuclear factor NF-кB pathway in inflammation. Cold Spring Harb. Perspect. Biol. 1:a001651. doi: 10.1101/cshperspect.a001651

Lebrun-Julien, F., and Di Polo, A. (2008). Molecular and cell-based approaches for neuroprotection in glaucoma. Optom. Vis. Sci. 85, 417-424. doi: 10.1097/OPX.0b013e31817841f7

Lebrun-Julien, F., Morquette, B., Douillette, A., Saragovi, H. U., and Di Polo, A. (2009). Inhibition of p75(NTR) in glia potentiates TrkA-mediated survival of injured retinal ganglion cells. Mol. Cell. Neurosci. 40, 410-420. doi: 10.1016/j.mcn.2008.12.005

Lee, J. Y., Kim, J. M., Ahn, J., Kim, H. J., Jeon, B. S., and Kim, T. W. (2014). Retinal nerve fiber layer thickness and visual hallucinations in Parkinson's Disease. Mov. Disord. 29, 61-67. doi: 10.1002/mds.25543

Lee, S., Van Bergen, N. J., Kong, G. Y., Chrysostomou, V., Waugh, H. S., O'neill, E. C., et al. (2011). Mitochondrial dysfunction in glaucoma and emerging bioenergetic therapies. Exp. Eye Res. 93, 204-212. doi: 10.1016/j.exer.2010.07.015

Linden, R., and Perry, V. H. (1983). Massive retinotectal projection in rats. Brain Res. 272, 145-149. doi: 10.1016/0006-8993(83)90371-2

Lu, M., Patsouris, D., Li, P., Flores-Riveros, J., Frincke, J. M., Watkins, S., et al. (2010). A new antidiabetic compound attenuates inflammation and insulin resistance in Zucker diabetic fatty rats. Am. J. Physiol. Endocrinol. Metab. 298, E1036-E1048. doi: 10.1152/ajpendo.00668.2009

Mac Nair, C. E., and Nickells, R. W. (2015). Neuroinflammation in glaucoma and optic nerve damage. Prog. Mol. Biol. Transl. Sci. 134, 343-363. doi: 10.1016/bs.pmbts.2015.06.010

Madeira, M. H., Boia, R., Santos, P. F., Ambrosio, A. F., and Santiago, A. R. (2015). Contribution of microglia-mediated neuroinflammation to retinal degenerative diseases. Mediators Inflamm. 2015:673090. doi: 10.1155/2015/ 673090

Matousek, S. B., Ghosh, S., Shaftel, S. S., Kyrkanides, S., Olschowka, J. A., and O'banion, M. K. (2012). Chronic IL-1beta-mediated neuroinflammation mitigates amyloid pathology in a mouse model of Alzheimer's disease without inducing overt neurodegeneration. J. Neuroimmune Pharmacol. 7, 156-164. doi: $10.1007 / \mathrm{s} 11481-011-9331-2$ 
Mattson, M. P., and Camandola, S. (2001). NF- $\mathrm{B}$ in neuronal plasticity and neurodegenerative disorders. J. Clin. Invest. 107, 247-254. doi: 10.1172/JCI11916

Mckinnon, S. J. (2012). The cell and molecular biology of glaucoma: common neurodegenerative pathways and relevance to glaucoma. Invest. Ophthalmol. Vis. Sci. 53, 2485-2487. doi: 10.1167/iovs.12-9483j

Meeker, R. B., and Williams, K. S. (2015). The p75 neurotrophin receptor: at the crossroad of neural repair and death. Neural Regen. Res. 10, 721-725. doi: 10.4103/1673-5374.156967

Mucke, L., Masliah, E., Johnson, W. B., Ruppe, M. D., Alford, M., Rockenstein, E. M., et al. (1994). Synaptotrophic effects of human amyloid beta protein precursors in the cortex of transgenic mice. Brain Res. 666, 151-167. doi: 10.1016/0006-8993(94)90767-6

Naylor, J. C., Hulette, C. M., Steffens, D. C., Shampine, L. J., Ervin, J. F., Payne, V. M., et al. (2008). Cerebrospinal fluid dehydroepiandrosterone levels are correlated with brain dehydroepiandrosterone levels, elevated in Alzheimer's disease, and related to neuropathological disease stage. J. Clin. Endocrinol. Metab. 93, 3173-3178. doi: 10.1210/jc.2007-1229

Nickells, R. W., Howell, G. R., Soto, I., and John, S. W. (2012). Under pressure: cellular and molecular responses during glaucoma, a common neurodegeneration with axonopathy. Annu. Rev. Neurosci. 35, 153-179. doi: 10.1146/annurev.neuro.051508.135728

Nicoletti, F., Philippens, I., Fagone, P., Ahlem, C. N., Reading, C. L., Frincke, J. M., et al. (2012). 17 $\alpha$-Ethynyl-androst-5-ene-3 $\beta, 7 \beta, 17 \beta$-triol (HE3286) Is neuroprotective and reduces motor impairment and neuroinflammation in a murine MPTP Model of Parkinson's Disease. Parkinsons Dis. 2012:969418. doi: 10.1155/2012/969418

Noguchi, Y., Shinozaki, Y., Fujishita, K., Shibata, K., Imura, Y., Morizawa, Y., et al. (2013). Astrocytes protect neurons against methylmercury via ATP/P2Y(1) receptor-mediated pathways in astrocytes. PLoS ONE 8:e57898. doi: 10.1371/journal.pone.0057898

Offner, H., Firestein, G. S., Boyle, D. L., Pieters, R., Frincke, J. M., Garsd, A., et al. (2009). An orally bioavailable synthetic analog of an active dehydroepiandrosterone metabolite reduces established disease in rodent models of rheumatoid arthritis. J. Pharmacol. Exp. Ther. 329, 1100-1109. doi: 10.1124/jpet.108.145086

Offner, H., Zamora, A., Drought, H., Matejuk, A., Auci, D. L., Morgan, E. E., et al. (2002). A synthetic androstene derivative and a natural androstene metabolite inhibit relapsing-remitting EAE. J. Neuroimmunol. 130, 128-139. doi: 10.1016/S0165-5728(02)00214-X

Quigley, H. A., and Broman, A. T. (2006). The number of people with glaucoma worldwide in 2010 and 2020. Br. J. Ophthalmol. 90, 262-267. doi: 10.1136/bjo.2005.081224

Rasmuson, S., Nasman, B., and Olsson, T. (2011). Increased serum levels of dehydroepiandrosterone (DHEA) and interleukin-6 (IL-6) in women with mild to moderate Alzheimer's disease. Int. Psychogeriatr. 23, 1386-1392. doi: $10.1017 /$ S1041610211000810

Razali, N., Agarwal, R., Agarwal, P., Kapitonova, M. Y., Kannan Kutty, M., Smirnov, A., et al. (2015). Anterior and posterior segment changes in rat eyes with chronic steroid administration and their responsiveness to antiglaucoma drugs. Eur. J. Pharmacol. 749, 73-80. doi: 10.1016/j.ejphar.2014. 11.029

Razeghinejad, M. R., and Katz, L. J. (2012). Steroid-induced iatrogenic glaucoma. Ophthalmic Res. 47, 66-80. doi: 10.1159/000328630

Reading, C. L., Flores-Riveros, J., Stickney, D. R., and Frincke, J. M. (2013a). An anti-inflammatory sterol decreases obesity-related inflammationinduced insulin resistance and metabolic dysregulation. Mediators Inflamm. 2013:814989. doi: 10.1155/2013/814989

Reading, C. L., Frincke, J. M., and White, S. K. (2012). Molecular targets for $17 \alpha$-ethynyl-5-androstene-3 $\beta, 7 \beta, 17 \beta$-triol, an anti-inflammatory agent derived from the human metabolome. PLoS ONE 7:e32147. doi: 10.1371/journal.pone.0032147

Reading, C. L., Stickney, D. R., Flores-Riveros, J., Destiche, D. A., Ahlem, C. N., Cefalu, W. T., et al. (2013b). A synthetic anti-inflammatory sterol improves insulin sensitivity in insulin-resistant obese impaired glucose tolerance subjects. Obesity 21, E343-E349. doi: 10.1002/oby.20207

Roh, M., Zhang, Y., Murakami, Y., Thanos, A., Lee, S. C., Vavvas, D. G., et al. (2012). Etanercept, a widely used inhibitor of tumor necrosis factor- $\alpha$ (TNF- $\alpha$ ), prevents retinal ganglion cell loss in a rat model of glaucoma. PLoS ONE 7:e40065. doi: 10.1371/journal.pone.0040065

Russo, R., Varano, G. P., Adornetto, A., Nucci, C., Corasaniti, M. T., Bagetta, G., et al. (2016). Retinal ganglion cell death in glaucoma: exploring the role of neuroinflammation. Eur. J. Pharmacol. 787, 134-142. doi: 10.1016/j.ejphar.2016.03.064

Saidha, S., Al-Louzi, O., Ratchford, J. N., Bhargava, P., Oh, J., Newsome, S. D., et al. (2015). Optical coherence tomography reflects brain atrophy in multiple sclerosis: a four-year study. Ann. Neurol. 78, 801-813. doi: 10.1002/ana.24487

Saleh, A., Roy Chowdhury, S. K., Smith, D. R., Balakrishnan, S., Tessler, L., Martens, C., et al. (2013). Ciliary neurotrophic factor activates NF- $\kappa$ B to enhance mitochondrial bioenergetics and prevent neuropathy in sensory neurons of streptozotocin-induced diabetic rodents. Neuropharmacology 65, 65-73. doi: 10.1016/j.neuropharm.2012.09.015

Samuel, M. A., Zhang, Y., Meister, M., and Sanes, J. R. (2011). Age-related alterations in neurons of the mouse retina. J. Neurosci. 31, 16033-16044. doi: 10.1523/JNEUROSCI.3580-11.2011

Sappington, R. M., and Calkins, D. J. (2008). Contribution of TRPV1 to microgliaderived IL-6 and NFKB translocation with elevated hydrostatic pressure. Invest. Ophthalmol. Vis. Sci. 49, 3004-3017. doi: 10.1167/iovs.07-1355

Sappington, R. M., Carlson, B. J., Crish, S. D., and Calkins, D. J. (2010). The microbead occlusion model: a paradigm for induced ocular hypertension in rats and mice. Invest. Ophthalmol. Vis. Sci. 51, 207-216. doi: 10.1167/iovs.09-3947

Sappington, R. M., Chan, M., and Calkins, D. J. (2006). Interleukin-6 protects retinal ganglion cells from pressure-induced death. Invest. Ophthalmol. Vis. Sci. 47, 2932-2942. doi: 10.1167/iovs.05-1407

Sappington, R. M., Sidorova, T., Long, D. J., and Calkins, D. J. (2009). TRPV1: contribution to retinal ganglion cell apoptosis and increased intracellular $\mathrm{Ca}^{2+}$ with exposure to hydrostatic pressure. Invest. Ophthalmol. Vis. Sci. 50, 717-728. doi: 10.1167/iovs.08-2321

Schneider, C. A., Rasband, W. S., and Eliceiri, K. W. (2012). NIH Image to ImageJ: 25 years of image analysis. Nat. Methods 9, 671-675. doi: 10.1038/nmeth.2089

Schober, M. S., Chidlow, G., Wood, J. P., and Casson, R. J. (2008). Bioenergeticbased neuroprotection and glaucoma. Clin. Exp. Ophthalmol. 36, 377-385. doi: 10.1111/j.1442-9071.2008.01740.x

Schubert, D., and Behl, C. (1993). The expression of amyloid beta protein precursor protects nerve cells from beta-amyloid and glutamate toxicity and alters their interaction with the extracellular matrix. Brain Res. 629, 275-282. doi: 10.1016/0006-8993(93)91331-L

Shimazawa, M., Ito, Y., Inokuchi, Y., Yamanaka, H., Nakanishi, T., Hayashi, T., et al. (2012). An alteration in the lateral geniculate nucleus of experimental glaucoma monkeys: in vivo positron emission tomography imaging of glial activation. PLoS ONE 7:e30526. doi: 10.1371/journal.pone.0030526

Sims, S. M., Holmgren, L., Cathcart, H. M., and Sappington, R. M. (2012). Spatial regulation of interleukin-6 signaling in response to neurodegenerative stressors in the retina. Am. J. Neurodegener. Dis. 1, 168-179.

Song, C., Zhang, Y., and Dong, Y. (2013). Acute and subacute IL-1 $\beta$ administrations differentially modulate neuroimmune and neurotrophic systems: possible implications for neuroprotection and neurodegeneration. J. Neuroinflammation 10:59. doi: 10.1186/1742-2094-10-59

Soto, I., and Howell, G. R. (2014). The complex role of neuroinflammation in glaucoma. Cold Spring Harb. Perspect. Med. 4:a017269. doi: 10.1101/cshperspect.a017269

Stasi, K., Nagel, D., Yang, X., Ren, L., Mittag, T., and Danias, J. (2007). Ceruloplasmin upregulation in retina of murine and human glaucomatous eyes. Invest. Ophthalmol. Vis. Sci. 48, 727-732. doi: 10.1167/iovs.06-0497

Stasi, K., Nagel, D., Yang, X., Wang, R. F., Ren, L., Podos, S. M., et al. (2006). Complement component 1Q (C1Q) upregulation in retina of murine, primate, and human glaucomatous eyes. Invest. Ophthalmol. Vis. Sci. 47, 1024-1029. doi: 10.1167/iovs.05-0830

Stevens, B., Allen, N. J., Vazquez, L. E., Howell, G. R., Christopherson, K. S., Nouri, N., et al. (2007). The classical complement cascade mediates CNS synapse elimination. Cell 131, 1164-1178. doi: 10.1016/j.cell.2007.10.036

Tezel, G., Li, L. Y., Patil, R. V., and Wax, M. B. (2001). TNF-alpha and TNF-alpha receptor-1 in the retina of normal and glaucomatous eyes. Invest. Ophthalmol. Vis. Sci. 42, 1787-1794.

Vidal-Sanz, M., Valiente-Soriano, F. J., Ortin-Martinez, A., Nadal-Nicolas, F. M., Jimenez-Lopez, M., Salinas-Navarro, M., et al. (2015). Retinal 
neurodegeneration in experimental glaucoma. Prog. Brain Res. 220, 1-35. doi: 10.1016/bs.pbr.2015.04.008

Wang, T., Villegas, S., Huang, Y., White, S. K., Ahlem, C., Lu, M., et al. (2010). Amelioration of glucose intolerance by the synthetic androstene HE3286: link to inflammatory pathways. J. Pharmacol. Exp. Ther. 333, 70-80. doi: 10.1124/jpet.109.161182

Wang, X., Sam-Wah Tay, S., and Ng, Y. K. (2000). Nitric oxide, microglial activities and neuronal cell death in the lateral geniculate nucleus of glaucomatous rats. Brain Res. 878, 136-147. doi: 10.1016/S0006-8993(00)02727-X

Ward, N. J., Ho, K. W., Lambert, W. S., Weitlauf, C., and Calkins, D. J. (2014). Absence of transient receptor potential vanilloid-1 accelerates stressinduced axonopathy in the optic projection. J. Neurosci. 34, 3161-3170. doi: 10.1523/JNEUROSCI.4089-13.2014

Weber, A. J., Viswanathan, S., Ramanathan, C., and Harman, C. D. (2010). Combined application of BDNF to the eye and brain enhances ganglion cell survival and function in the cat after optic nerve injury. Invest. Ophthalmol. Vis. Sci. 51, 327-334. doi: 10.1167/iovs.09-3740

Wei, Y., Wang, N., Lu, Q., Zhang, N., Zheng, D., and Li, J. (2007). Enhanced protein expressions of sortilin and p75NTR in retina of rat following elevated intraocular pressure-induced retinal ischemia. Neurosci. Lett. 429, 169-174. doi: 10.1016/j.neulet.2007.10.012

Weitlauf, C., Ward, N. J., Lambert, W. S., Sidorova, T. N., Ho, K. W., Sappington, R. M., et al. (2014). Short-term increases in transient receptor potential vanilloid-1 mediate stress-induced enhancement of neuronal excitation. J. Neurosci. 34, 15369-15381. doi: 10.1523/JNEUROSCI.3424-14.2014

Wilson, G. N., Inman, D. M., Dengler Crish, C. M., Smith, M. A., and Crish, S. D. (2015). Early pro-inflammatory cytokine elevations in the DBA/2J mouse model of glaucoma. J. Neuroinflammation 12, 176 doi: 10.1186/s12974-015-0399-0

Yankner, B. A., Dawes, L. R., Fisher, S., Villa-Komaroff, L., Oster-Granite, M. L., and Neve, R. L. (1989). Neurotoxicity of a fragment of the amyloid precursor associated with Alzheimer's disease. Science 245, 417-420. doi: $10.1126 /$ science. 2474201

Yuan, L., and Neufeld, A. H. (2001). Activated microglia in the human glaucomatous optic nerve head. J. Neurosci. Res. 64, 523-532. doi: $10.1002 /$ jnr.1104

Zheng, H., and Koo, E. H. (2011). Biology and pathophysiology of the amyloid precursor protein. Mol. Neurodegener. 6:27. doi: 10.1186/17501326-6-27

Conflict of Interest Statement: The authors declare that this research was supported by a grant from Harbor Therapeutics, Inc (San Diego, CA).

The reviewer $\mathrm{MH}$ and handling Editor declared their shared affiliation, and the handling Editor states that the process nevertheless met the standards of a fair and objective review.

Copyright (C) 2017 Lambert, Carlson, Formichella, Sappington, Ahlem and Calkins. This is an open-access article distributed under the terms of the Creative Commons Attribution License (CC BY). The use, distribution or reproduction in other forums is permitted, provided the original author(s) or licensor are credited and that the original publication in this journal is cited, in accordance with accepted academic practice. No use, distribution or reproduction is permitted which does not comply with these terms. 